\title{
A DISCRETE EXTENSION OF THE BLASCHKE ROLLING BALL THEOREM
}

\author{
SZILÁRD GY. RÉVÉSZ
}

\begin{abstract}
The Rolling Ball Theorem asserts that given a convex body $K \subset \mathbb{R}^{d}$ in Euclidean space and having a $C^{2}$-smooth surface $\partial K$ with all principal curvatures not exceeding $c>0$ at all boundary points, $K$ necessarily has the property that to each boundary point there exists a ball $B_{r}$ of radius $r=1 / c$, fully contained in $K$ and touching $\partial K$ at the given boundary point from the inside of $K$.

In the present work we prove a discrete analogue of the result on the plane. We consider a certain discrete condition on the curvature, namely that to any boundary points $\mathbf{x}, \mathbf{y} \in \partial K$ with $|\mathbf{x}-\mathbf{y}| \leq \tau$, the angle $\varphi\left(\mathbf{n}_{\mathbf{x}}, \mathbf{n}_{\mathbf{y}}\right):=\arccos \left\langle\mathbf{n}_{\mathbf{x}}, \mathbf{n}_{\mathbf{y}}\right\rangle$ of any unit outer normals $\mathbf{n}_{\mathbf{x}}, \mathbf{n}_{\mathbf{y}}$ at $\mathbf{x}$ and at $\mathbf{y}$, resp., does not exceed a given angle $\varphi$. Then we construct a corresponding body, $M(\tau, \varphi)$, which is to lie fully within $K$ while containing the given boundary point $\mathbf{x} \in \partial K$.

In dimension $d=2$, that is, on the plane, $M$ is almost a regular $n$-gon, and the result allows to recover the precise form of Blaschke's Rolling Ball Theorem in the limit.

Similarly, we consider the dual type discrete Blaschke theorems ensuring certain circumscribed polygons. In the limit, the discrete theorem enables us to provide a new proof for a strong result of Strantzen assuming only a.e. existence and lower estimations on the curvature.

For $d \geq 3$, directly we can derive only a weaker, quasi-precise form of the discrete inscribed ball theorem, while no space version of the circumscribed ball theorem is found. However, at least the higher dimensional smooth cases follow already from the plane versions of the smooth theorems, which obtain as limiting cases also from our discrete versions.
\end{abstract}

\section{INTRODUCTION}

Let $\mathbb{R}^{d}$ be the usual Euclidean space of dimension $d$, equipped with the Euclidean distance $|\cdot|$. Our starting point is the following classical result of Blaschke [1, p. 116].

Theorem A. (Blaschke). Assume that the convex domain $K \subset \mathbb{R}^{2}$ has $C^{2}$ boundary $\Gamma=\partial K$ and that with the positive constant $\kappa_{0}>0$ the curvature satisfies $\kappa(\mathbf{z}) \leq \kappa_{0}$ at all boundary points $\mathbf{z} \in \Gamma$. Then to each boundary points $\mathbf{z} \in \Gamma$ there exists a disk $D_{R}$ of radius $R=1 / \kappa_{0}$, such that $\mathbf{z} \in \partial D_{R}$, and $D_{R} \subset K$.

Note that the result, although seemingly local, does not allow for extensions to nonconvex curves $\Gamma$. One can draw pictures of leg-bone like shapes of arbitrarily small upper bound of (positive) curvature, while at some points of touching containing arbitrarily small disks only. The reason is that the curve, after starting off from a certain boundary point $\mathbf{x}$, and then leaning back a bit, can eventually return arbitrarily close to the point from where it started: hence a prescribed size of disk cannot be inscribed.

On the other hand the Blaschke Theorem extends to any dimension $d \in \mathbb{N}$. Also, the result has a similar, dual version, too, see [1, p. 116].

Supported in part by the Hungarian National Foundation for Scientific Research, Project \#s K-61908 and K-72731. 
Theorem B. (Blaschke.) Assume that $K \subset \mathbb{R}^{2}$ is a convex domain with $C^{2}$-smooth boundary curve $\gamma$ having curvature $\kappa \geq \kappa_{0}$ all over $\gamma$. Then to all boundary point $\mathbf{x} \in \gamma$ there exists a disk $D_{R}$ of radius $R=1 / \kappa_{0}$, such that $\mathbf{x} \in \partial D_{R}$, and $K \subset D_{R}$.

In Section 2 we introduce a few notions and recall auxiliary facts. In $\S 3$ we formulate and prove the two basic results - the discrete forms of the Blaschke Theorems - of our paper. Then we show how our discrete approach yields a new, straightforward proof for a more involved sharpening of Theorem A, originally due to Strantzen.

This all concerns dimension 2. Only in Section 5 will we consider the case of higher dimensional Euclidean spaces. Certain corresponding results hold also in $\mathbb{R}^{d}$, but they are less satisfactory, as the classical Blaschke theorem cannot be recovered from them in the limit. Nevertheless, it is worthy to formulate them, in view of certain applications in multivariate approximation, what we have in mind when analyzing these questions.

\section{Preliminaries, Geometrical notions}

Recall that the term planar convex body stands for a compact, convex subset of $\mathbb{C} \cong \mathbb{R}^{2}$ having nonempty interior. For a (planar) convex body $K$ any interior point $z$ defines a parametrization $\gamma(\varphi)$ - the usual polar coordinate representation of the boundary $\partial K$, - taking the unique point $\left\{z+t e^{i \varphi}: t \in(0, \infty)\right\} \cap \partial K$ for the definition of $\gamma(\varphi)$. This defines the closed Jordan curve $\Gamma=\partial K$ and its parametrization $\gamma:[0,2 \pi] \rightarrow \mathbb{C}$. By convexity, from any boundary point $\zeta=\gamma(\theta) \in \partial K$, locally the chords to boundary points with parameter $<\theta$ or with $>\theta$ have arguments below and above the argument of the direction of any supporting line at $\zeta$. Thus the tangent direction or argument function $\alpha_{-}(\theta)$ can be defined as e.g. the supremum of arguments of chords from the left; similarly, $\alpha_{+}(\theta):=\inf \{\arg (z-\zeta): z=\gamma(\varphi), \varphi>\theta\}$, and any line $\zeta+e^{i \beta} \mathbb{R}$ with $\alpha_{-}(\theta) \leq \beta \leq \alpha_{+}(\theta)$ is a supporting line to $K$ at $\zeta=\gamma(\theta) \in \partial K$. In particular the curve $\gamma$ is differentiable at $\zeta=\gamma(\theta)$ if and only if $\alpha_{-}(\theta)=\alpha_{+}(\theta)$; in this case the tangent of $\gamma$ at $\zeta$ is $\zeta+e^{i \alpha} \mathbb{R}$ with the unique value of $\alpha=\alpha_{-}(\theta)=\alpha_{+}(\theta)$. It is clear that interpreting $\alpha_{ \pm}$ as functions on the boundary points $\zeta \in \partial K$, we obtain a parametrization-independent function. In other words, we are allowed to change parameterizations to arc length, say, when in case of $|\Gamma|=\ell(|\Gamma|$ meaning the length of $\Gamma:=\partial K)$ the functions $\alpha_{ \pm}$map $[0, \ell]$ to $[0,2 \pi]$.

Observe that $\alpha_{ \pm}$are nondecreasing functions with total variation $\operatorname{Var}\left[\alpha_{ \pm}\right]=2 \pi$, and that they have a common value precisely at continuity points, which occur exactly at points where the supporting line to $K$ is unique. At points of discontinuity $\alpha_{ \pm}$is the left-, resp. right continuous extension of the same function. For convenience, and for better matching with [3], we may even define the function $\alpha:=\left(\alpha_{+}+\alpha_{-}\right) / 2$ all over the parameter interval.

For obvious geometric reasons we call the jump function $\beta:=\alpha_{+}-\alpha_{-}$the supplementary angle function. In fact, $\beta$ and the usual Lebesgue decomposition of the nondecreasing function $\alpha_{+}$to $\alpha_{+}=\sigma+\alpha_{*}+\alpha_{0}$, consisting of the pure jump function $\sigma$, the nondecreasing singular component $\alpha_{*}$, and the absolute continuous part $\alpha_{0}$, are closely related. By monotonicity there are at most countable many points where $\beta(x)>0$, and in view of bounded variation we even have $\sum_{x} \beta(x) \leq 2 \pi$, hence the definition $\mu:=\sum_{x} \beta(x) \delta_{x}$ defines a bounded, non-negative Borel measure on $[0,2 \pi)$. Now it is clear that $\sigma(x)=$ $\mu([0, x])$, while $\alpha_{*}^{\prime}=0$ a.e., and $\alpha_{0}$ is absolutely continuous. In particular, $\alpha$ or $\alpha_{+}$is differentiable at $x$ provided that $\beta(x)=0$ and $x$ is not in the exceptional set of nondifferentiable points with respect to $\alpha_{*}$ or $\alpha_{0}$. That is, we have differentiability almost 
everywhere, and

$$
\begin{aligned}
& \int_{x}^{y} \alpha^{\prime}=\alpha_{0}(y)-\alpha_{0}(x)=\lim _{z \rightarrow x-0} \alpha_{0}(y)-\alpha_{0}(z) \\
&\left.=\lim _{z \rightarrow x-0}\left\{\left[\alpha_{+}(y)-\sigma(y)-\alpha_{*}(y)\right)\right]-\left[\alpha_{+}(z)-\sigma(z)-\alpha_{*}(z)\right]\right\} \\
&(1) \quad=\alpha_{+}(y)-\beta(y)-\mu([x, y))-\lim _{z \rightarrow x-0} \alpha_{+}(z)-\lim _{z \rightarrow x-0}\left[\alpha_{*}(y)-\alpha_{*}(z)\right] \leq \alpha_{-}(y)-\alpha_{+}(x) .
\end{aligned}
$$

It follows that

$$
\alpha^{\prime}(t) \geq \lambda \quad \text { a.e. } t \in[0, a]
$$

holds true if and only if we have

$$
\alpha_{ \pm}(y)-\alpha_{ \pm}(x) \geq \lambda(y-x) \quad \forall x, y \in[0, a] .
$$

Here we restricted ourselves to the arc length parametrization taken in positive orientation. Recall that one of the most important geometric quantities, curvature, is just $\kappa(s):=$ $\alpha^{\prime}(s)$, whenever parametrization is by arc length $s$.

Thus we can rewrite (2) as

$$
\kappa(t) \geq \lambda \quad \text { a.e. } \quad t \in[0, a],
$$

or, with radius of curvature $\rho(t):=1 / \kappa(t)$ introduced (writing $1 / 0=\infty$ ),

$$
\rho(t) \leq \frac{1}{\lambda} \quad \text { a.e. } \quad t \in[0, a]
$$

Again, $\rho$ is a parametrization-invariant quantity (describing the radius of the osculating circle). Actually, it is easy to translate all these conditions to arbitrary parametrization of the tangent angle function $\alpha$. Since also curvature and radius of curvature are parametrization-invariant quantities, all the above hold for any parametrization.

Moreover, with a general parametrization let $|\Gamma(\eta, \zeta)|$ stand for the length of the counterclockwise arc $\Gamma(\eta, \zeta)$ of the rectifiable Jordan curve $\Gamma$ between the two points $\zeta, \eta \in \Gamma=\partial K$. We can then say that the curve satisfies a Lipschitz-type increase or subdifferential condition whenever

$$
\left|\alpha_{ \pm}(\eta)-\alpha_{ \pm}(\zeta)\right| \geq \lambda|\Gamma(\eta, \zeta)| \quad(\forall \zeta, \eta \in \Gamma)
$$

here meaning by $\alpha_{ \pm}(\xi)$, for $\xi \in \Gamma$, not values in $[0,2 \pi)$, but a locally monotonously increasing branch of $\alpha_{ \pm}$, with jumps in $(0, \pi)$, along the counterclockwise $\operatorname{arc} \Gamma(\eta, \zeta)$ of $\Gamma$. Clearly, the above considerations show that all the above are equivalent.

In the paper we use the notation $\alpha$ (and also $\alpha_{ \pm}$) for the tangent angle, $\kappa$ for the curvature, and $\rho$ for the radius of curvature. The counterclockwise taken right hand side tangent unit vector(s) will be denoted by $\mathbf{t}$, and the outer unit normal vectors by $\mathbf{n}$. These notations we will use basically in function of the arc length parametrization $s$, but with a slight abuse of notation also $\alpha_{-}(\varphi), \mathbf{t}(\mathbf{x}), \mathbf{n}(\mathbf{x})$ etc. may occur with the obvious meaning.

Note that $\mathbf{t}(\mathbf{x})=i \mathbf{n}(\mathbf{x}))$ and also $\mathbf{t}(\mathbf{x})=\dot{\gamma}(s)$ when $\mathbf{x}=\mathbf{x}(s) \in \gamma$ and the parametrization/differentiation, symbolized by the dot, is with respect to arc length; moreover, with $\nu(s): \arg (\mathbf{n}(\mathbf{x}(s))$ we obviously have $\alpha \equiv \nu+\pi / 2 \bmod 2 \pi$ at least at points of continuity of $\alpha$ and $\nu$. To avoid mod $2 \pi$ equality, we can shift to the universal covering spaces and maps and consider $\widetilde{\alpha}, \widetilde{\nu}$, i.e. $\widetilde{\mathbf{t}}, \widetilde{\mathbf{n}}-$ e.g. in case of $\widetilde{\mathbf{n}}$ we will somewhat detail this right below. However, note a slight difference in handling $\alpha$ and $\widetilde{\mathbf{n}}$ : the first is taken as a singlevalued function, with values $\alpha(s):=\frac{1}{2}\left\{\alpha_{-}(s)+\alpha_{+}(s)\right\}$ at points of discontinuity, while $\widetilde{\mathbf{n}}$ is a multivalued function attaining a full closed interval $\left[\widetilde{\mathbf{n}}_{-}(s), \widetilde{\mathbf{n}}_{+}(s)\right]$ whenever $s$ is a point of discontinuity. Also recall that curvature, whenever it exists, is $|\ddot{\gamma}(s)|=\alpha^{\prime}(s)=\widetilde{\mathbf{n}}^{\prime}(s)$. 
In this work we mean by a multi-valued function $\Phi$ from $X$ to $Y$ a (non-empty-valued) mapping $\Phi: X \rightarrow 2^{Y} \backslash\{\emptyset\}$, i.e. we assume that the domain of $\Phi$ is always the whole of $X$ and that $\emptyset \neq \Phi(x) \subset Y$ for all $x \in X$. Recall the notions of modulus of continuity and minimal oscillation in the full generality of multi-valued functions between metric spaces.

Definition 1 (modulus of continuity and minimal oscillation). Let $\left(X, d_{X}\right)$ and $\left(Y, d_{Y}\right)$ be metric spaces. We call the modulus of continuity of the multivalued function $\Phi$ from $X$ to $Y$ the quantity

$$
\omega(\Phi, \tau):=\sup \left\{d_{Y}\left(y, y^{\prime}\right): x, x^{\prime} \in X, d_{X}\left(x, x^{\prime}\right) \leq \tau, y \in \Phi(x), y^{\prime} \in \Phi\left(x^{\prime}\right)\right\} .
$$

Similarly, we call minimal oscillation of $\Phi$ the quantity

$$
\Omega(\Phi, \tau):=\inf \left\{d_{Y}\left(y, y^{\prime}\right): x, x^{\prime} \in X, d_{X}\left(x, x^{\prime}\right) \geq \tau, y \in \Phi(x), y^{\prime} \in \Phi\left(x^{\prime}\right)\right\} .
$$

If we are given a multi-valued unit vector function $\mathbf{v}(\mathbf{x}): H \rightarrow 2^{S^{d-1}} \backslash\{\emptyset\}$, where $H \subset \mathbb{R}^{d}$ and $S^{d-1}$ is the unit ball of $\mathbb{R}^{d}$, then the derived formulae become:

(7) $\omega(\tau):=\omega(\mathbf{v}, \tau):=\sup \{\arccos \langle\mathbf{u}, \mathbf{w}\rangle: \mathbf{x}, \mathbf{y} \in H,|\mathbf{x}-\mathbf{y}| \leq \tau, \mathbf{u} \in \mathbf{v}(\mathbf{x}), \mathbf{w} \in \mathbf{v}(\mathbf{y})\}$, and

(8) $\Omega(\tau):=\Omega(\mathbf{v}, \tau):=\inf \{\arccos \langle\mathbf{u}, \mathbf{w}\rangle: \mathbf{x}, \mathbf{y} \in H,|\mathbf{x}-\mathbf{y}| \geq \tau, \mathbf{u} \in \mathbf{v}(\mathbf{x}), \mathbf{w} \in \mathbf{v}(\mathbf{y})\}$.

For a planar multi-valued unit vector function $\mathbf{v}: H \rightarrow 2^{S^{1}} \backslash\{\emptyset\}$, where $H \subset \mathbb{R}^{2} \simeq \mathbb{C}$ and $S^{1}$ is the unit circle in $\mathbb{R}^{2}$, we can parameterize the unit circle $S^{1}$ by the corresponding angle $\varphi$ and thus write $\mathbf{v}(\mathbf{x})=e^{i \Phi(\mathbf{x})}$ with $\Phi(\mathbf{x}):=\arg (\mathbf{v}(\mathbf{x}))$ being the corresponding angle. We will somewhat elaborate on this observation in the case when our multi-valued vector function is the outward normal vector(s) function $\mathbf{n}(\mathbf{x})$ of a closed convex curve.

Let $\gamma$ be the boundary curve of a convex body in $\mathbb{R}^{2}$, which will be considered as oriented counterclockwise, and let the multivalued function $\mathbf{n}(\mathbf{x}): \gamma \rightarrow 2^{S^{1}} \backslash\{\emptyset\}$ be defined as the set of all outward unit normal vectors of $\gamma$ at the point $\mathbf{x} \in \gamma$. Observe that the set $\mathbf{n}(\mathbf{x})$ of the set of values of $\mathbf{n}$ at any $\mathbf{x} \in \gamma$ is either a point, or a closed segment of length less than $\pi$. Then there exists a unique lifting $\tilde{\mathbf{n}}$ of $\mathbf{n}$ from the universal covering space $\tilde{\gamma}\left(\simeq \mathbb{R}\right.$, see below) of $\gamma$ to the universal covering space $\mathbb{R}=\tilde{S}^{1}$ of $S^{1}$, with the respective universal covering maps $\pi_{\gamma}: \tilde{\gamma} \rightarrow \gamma$ and $\pi_{S^{1}}: \tilde{S}^{1} \rightarrow S^{1}$, with properties to be described below. Here we do not want to recall the concept of the universal covering spaces from algebraic topology in its generality, but restrict ourselves to give it in the situation described above. As already said, $\tilde{S}^{1}=\mathbb{R}$ and the corresponding universal covering map is $\pi_{S^{1}}: x \rightarrow(\cos x, \sin x)$ (We consider, as usual, $S^{1}$ as $\mathbb{R} \bmod 2 \pi$.) Similarly, for $\gamma$ we have $\tilde{\gamma}=\mathbb{R}$, with universal covering map $\pi_{\gamma}: \mathbb{R} \rightarrow \gamma$ given in the following way. Let us fix some arbitrary point $\mathbf{x}_{\mathbf{0}} \in \gamma$, (the following considerations will be independent of $\mathbf{x}_{\mathbf{0}}$, in the natural sense). Let us denote by $\ell$ the length of $\gamma$. Then for $\lambda \in \mathbb{R}=\tilde{\gamma}$ we have that $\pi_{\gamma}(\lambda) \in \gamma$ is that unique point $\mathbf{x}$ of $\gamma$, for which the counterclockwise measured arc $\mathbf{x}_{\mathbf{0}} \mathbf{x}$ has a length $\lambda \bmod \ell$.

Now we describe the postulates for the multivalued function $\tilde{\mathbf{n}}: \mathbb{R}=\tilde{\gamma} \rightarrow \tilde{S}^{1}=\mathbb{R}$, which determine it uniquely. First of all, we must have the equality $\pi_{S^{1}} \circ \tilde{\mathbf{n}}=\mathbf{n} \circ \pi_{\gamma}$, where $\circ$ denotes the composition of two multivalued functions. (In algebraic topology this is called commutativity of a certain square of mappings.) Second, the values of $\tilde{\mathbf{n}}$ must be either points or non-degenerate closed intervals (of length less than $\pi$; however this last property follows from the other ones). Third, $\tilde{\mathbf{n}}$ must be non-decreasing in the following sense: for $\lambda_{1}, \lambda_{2} \in \mathbb{R}, \lambda_{1}<\lambda_{2}$ we have $r_{1} \in \tilde{\mathbf{n}}\left(\lambda_{1}\right), r_{2} \in \tilde{\mathbf{n}}\left(\lambda_{2}\right) \Longrightarrow r_{1} \leq r_{2}$. Further, $\tilde{\mathbf{n}}$ must be a non-decreasing multivalued function, continuous from the left, i.e., for any $\lambda \in \mathbb{R}$ we have that for any $\varepsilon>0$ there exists a $\delta>0$, such that $\cup_{\mu \in(\lambda-\delta, \lambda)} \tilde{\mathbf{n}}(\mu) \subset$ 
$(\min \tilde{\mathbf{n}}(\lambda)-\varepsilon, \min \tilde{\mathbf{n}}(\lambda))$. Analogously, $\tilde{\mathbf{n}}$ must be a non-decreasing multi-valued function continuous from the right, i.e., for any $\lambda \in \mathbb{R}$ we have that for any $\varepsilon>0$ there exists a $\delta>0$, such that $\cup_{\mu \in(\lambda, \lambda+\delta)} \tilde{\mathbf{n}}(\mu) \subset(\max \tilde{\mathbf{n}}(\lambda), \max \tilde{\mathbf{n}}(\lambda)+\varepsilon)$. These are all the postulates for the multi-valued function $\tilde{\mathbf{n}}$. It is clear, that $\tilde{\mathbf{n}}$ exists and is uniquely determined, for fixed $\mathbf{x}_{\mathbf{0}}$ (and, for $\mathbf{x}_{\mathbf{0}}$ arbitrary, only the parametrization of $\mathbb{R}=\tilde{\gamma}$ changes, by a translation.)

The above listed properties imply still one important property of the multi-valued function $\tilde{\mathbf{n}}$ : we have for any $\lambda \in \mathbb{R}$ that $\tilde{\mathbf{n}}(\lambda+\ell)=\tilde{\mathbf{n}}(\lambda)+2 \pi$.

Definition 2. We define the modulus of continuity of the multi-valued normal vector function $\mathbf{n}(\mathbf{x})$ with respect to arc length as the (ordinary) modulus of continuity of the multi-valued lift-up function $\tilde{\mathbf{n}}: \mathbb{R} \rightarrow \mathbb{R} \backslash\{\emptyset\}$, i.e. as

$$
\tilde{\omega}(\tau):=\tilde{\omega}(\mathbf{n}, \tau):=\omega(\tilde{\mathbf{n}}, \tau)
$$

$$
:=\sup \left\{\left|r_{1}-r_{2}\right|\left|r_{1} \in \tilde{\mathbf{n}}\left(\lambda_{1}\right), r_{2} \in \tilde{\mathbf{n}}\left(\lambda_{2}\right), \lambda_{1}, \lambda_{2} \in \mathbb{R},\right| \lambda_{1}-\lambda_{2} \mid \leq \tau\right\} .
$$

Similarly, we define the minimal oscillation of the multi-valued normal vector function $\mathbf{n}(\mathbf{x})$ with respect to arc length as the (ordinary) minimal oscillation function of $\tilde{\mathbf{n}}$, i.e. as

$$
\begin{aligned}
\tilde{\Omega}(\tau):=\tilde{\Omega}(\mathbf{n}, \tau) & :=\Omega(\tilde{\mathbf{n}}, \tau) \\
& :=\inf \left\{\left|r_{1}-r_{2}\right|\left|r_{1} \in \tilde{\mathbf{n}}\left(\lambda_{1}\right), r_{2} \in \tilde{\mathbf{n}}\left(\lambda_{2}\right), \lambda_{1}, \lambda_{2} \in \mathbb{R},\right| \lambda_{1}-\lambda_{2} \mid \geq \tau\right\} .
\end{aligned}
$$

By writing "modulus of continuity" we do not mean to say anything like continuity of $\tilde{\mathbf{n}}$. In fact, if for some $\lambda \in \mathbb{R} \tilde{\mathbf{n}}(\lambda)$ is a non-degenerate closed segment, then the left-hand side and right-hand side limits of $\tilde{\mathbf{n}}$ at $\lambda$ - in the sense of the definition of continuity from the left or right, respectively - are surely different.

We evidently have that the modulus of continuity of $\tilde{\mathbf{n}}$ is subadditive, meaning $\tilde{\omega}\left(\tau_{1}+\right.$ $\left.\tau_{2}\right) \leq \tilde{\omega}\left(\tau_{1}\right)+\tilde{\omega}\left(\tau_{2}\right)$, and similarly, that the minimal oscillation of $\tilde{\mathbf{n}}$ is superadditive, meaning $\tilde{\Omega}\left(\tau_{1}+\tau_{2}\right) \geq \tilde{\Omega}\left(\tau_{1}\right)+\tilde{\Omega}\left(\tau_{2}\right)$. In fact, a standard property of the modulus of continuity of any (non-empty valued) multivalued function from $\mathbb{R}$ (or from any convex set, in the sense of metric intervals) to $\mathbb{R}$ is subadditivity, and similarly, minimal oscillation of such a function is superadditive. These properties with non-negativity and non-decreasing property also imply that $\tilde{\omega}(\tau) / \tau$ and $\tilde{\Omega}(\tau) / \tau$ have limits when $\tau \rightarrow 0$; moreover, $\lim _{\tau \rightarrow 0} \tilde{\omega}(\tau) / \tau=\sup \tilde{\omega}(\tau) / \tau$ and $\lim _{\tau \rightarrow 0} \tilde{\Omega}(\tau) / \tau=\inf \tilde{\Omega}(\tau) / \tau$. Note that metric convexity is essential here, so e.g. it is not clear if in $\mathbb{R}^{d}$ any proper analogy could be established.

Observe that if the curvature of $\gamma$ exists at $\mathbf{x}_{0}$, then for the non-empty valued multivalued function $\mathbf{n}(\mathbf{x}):=$ "set of values of all outer unit normal vectors of $\gamma$ at $\mathbf{x}$ ", we necessarily have $\# \mathbf{n}\left(\mathbf{x}_{0}\right)=1$ and the curvature can be written as

$$
\kappa\left(\mathbf{x}_{0}\right)=\lim _{\mathbf{y} \rightarrow \mathbf{x}_{0}} \frac{\arccos \langle\mathbf{n}(\mathbf{n}(\mathbf{y})}{\left.\left.\mid \mathbf{x}_{0}\right), \mathbf{v}\right\rangle},
$$

where the limit in (11) exists with arbitrary choice of $\mathbf{v} \in \mathbf{y}$ and is independent of this choice.

The next two propositions are well-known.

Proposition 1. Let $\gamma$ be a planar convex curve. Recall that (7) and (8) is the modulus of continuity and the minimal oscillation of the multi-valued normal vector function $\mathbf{n}(\mathbf{x})$ with respect to chord length, and that (9) and (10) stand for the modulus of continuity and the minimal oscillation of $\mathbf{n}(\mathbf{x})$ with respect to arc length. Then for all $\mathbf{x} \in \gamma$ with 
curvature $\kappa(\mathbf{x}) \in[0, \infty]$ we have

$$
\lim _{\tau \rightarrow 0} \frac{\Omega(\tau)}{\tau}=\lim _{\tau \rightarrow 0} \frac{\tilde{\Omega}(\tau)}{\tau} \leq \kappa(\mathbf{x}) \leq \lim _{\tau \rightarrow 0} \frac{\tilde{\omega}(\tau)}{\tau}=\lim _{\tau \rightarrow 0} \frac{\omega(\tau)}{\tau} .
$$

Proof. First of all, by definition and the obvious fact that chord length does not exceed arc length, it follows that $\Omega(\tau) \leq \tilde{\Omega}(\tau) \leq \tilde{\omega}(\tau) \leq \omega(\tau)$. We have already remarked, that the limits $\lim _{\tau \rightarrow 0} \tilde{\Omega}(\tau) / \tau$ and $\lim _{\tau \rightarrow 0} \tilde{\omega}(\tau) / \tau$ exist; moreover, $\lim _{\tau \rightarrow 0} \tilde{\Omega}(\tau) / \tau=\inf \tilde{\Omega}(\tau) / \tau \leq$ $2 \pi / \ell(\gamma)$ is necessarily finite.

On the other hand, let $\tau$ be any fixed value, chosen sufficiently small, and choose $0 \leq s<t<\ell(\gamma), \gamma(s)=\mathbf{x}$ and $\gamma(t)=\mathbf{y}$ with $|\mathbf{x}-\mathbf{y}|=\tau$ such that $\Omega(\tau)=\arccos \langle\mathbf{u}, \mathbf{v}\rangle$ with some $\mathbf{u} \in \mathbf{n}(\mathbf{x}), \mathbf{v} \in \mathbf{n}(\mathbf{y})$. Then clearly $\arg \mathbf{u}=\tilde{\mathbf{n}}_{+}(s), \arg \mathbf{v}=\tilde{\mathbf{n}}_{-}(t)$, also $\Omega(\tau)=$ $\tilde{\mathbf{n}}_{-}(t)-\tilde{\mathbf{n}}_{+}(s)$, and for all $s<\sigma<t$ we have $\tilde{\mathbf{n}}(\sigma) \subset\left[\tilde{\mathbf{n}}_{+}(s), \tilde{\mathbf{n}}_{-}(t)\right]$. Moreover, putting $\nu$ for the normal vector of the chord $\mathbf{y}-\mathbf{x}$, having right angle with it in the clockwise direction, we also have $\arg (\nu) \in\left[\tilde{\mathbf{n}}_{+}(s), \tilde{\mathbf{n}}_{-}(t)\right]$ because $\mathbf{y}-\mathbf{x}=\int_{s}^{t} \mathbf{t}(\sigma) d \sigma=\int_{s}^{t} i \mathbf{n}(\sigma) d \sigma$, and thus $\arg (\mathbf{y}-\mathbf{x}) \in\left[\tilde{\mathbf{n}}_{+}(s)+\pi / 2, \tilde{\mathbf{n}}_{-}(t)+\pi / 2\right] \bmod 2 \pi$.

Now we compare arc length and chord length. We find $\tau=|\mathbf{y}-\mathbf{x}|=\int_{s}^{t}\langle\mathbf{n}(\sigma), \nu\rangle d \sigma \geq$ $(s-t) \cos \left(\tilde{\mathbf{n}}_{-}(t)-\tilde{\mathbf{n}}_{+}(s)\right)=(s-t) \cos \Omega(\tau)$, and, as $\Omega(\tau)=O(\tau)$, we surely have $\cos (\Omega(\tau)) \rightarrow 1$ when $\tau \rightarrow 0$. It is also clear that $t-s \rightarrow 0$ together with $\tau \rightarrow 0$, so for $\tau$ chosen sufficiently small,

$$
\frac{\Omega(\tau)}{\tau} \geq \frac{\tilde{\Omega}(t-s)}{\tau}=\frac{t-s}{\tau} \frac{\tilde{\Omega}(t-s)}{t-s} \geq \frac{t-s}{\tau}(1-\varepsilon) \lim _{\xi \rightarrow 0} \frac{\tilde{\Omega}(\xi)}{\xi} \geq(1-\varepsilon)^{2} \lim _{\xi \rightarrow 0} \frac{\tilde{\Omega}(\xi)}{\xi}
$$

and it follows that the two limits of the oscillation functions coincide.

For the modulus of continuity type quantities note that if $\mathbf{n}$ is really multivalued, i.e. there exists some point $\mathbf{x} \in \gamma$ where $\mathbf{n}(\mathbf{x})$ consists of more than one vector, then $\tilde{\mathbf{n}}$ attains some closed interval and $\tilde{\omega}(\tau)$ does not go to 0 with $\tau$ : whence the arising limits must be $+\infty$. Therefore, it suffices to consider the case when $\mathbf{n}$, i.e. $\tilde{\mathbf{n}}$, are single-valued (and thus $\tilde{\mathbf{n}}$ is monotonous and continuous) functions.

Again, consider a given value $\tau>0$, sufficiently small, and a pair of extremal points $\mathbf{x}=\gamma(s)$ and $\mathbf{y}=\gamma(t)$ with $0 \leq s<t<\ell(\gamma)$ such that $\tau=|\mathbf{y}-\mathbf{x}|$ and $\omega(\tau)=$ $\arccos \langle\mathbf{n}(\mathbf{x}), \mathbf{n}(\mathbf{y})\rangle=\tilde{\mathbf{n}}(t)-\tilde{\mathbf{n}}(s)$. As above, for all $s<\sigma<t$ we have $\tilde{\mathbf{n}}(\sigma) \subset[\tilde{\mathbf{n}}(s), \tilde{\mathbf{n}}(t)]$. Moreover, $\tau=|\mathbf{y}-\mathbf{x}|=\int_{s}^{t}\langle\mathbf{n}(\sigma), \nu\rangle d \sigma \geq(t-s) \cos (\tilde{\mathbf{n}}(t)-\tilde{\mathbf{n}}(s))=(t-s) \cos \omega(\tau)$, and, as $\omega(\tau) \rightarrow 0$, we surely have $\cos (\omega(\tau)) \rightarrow 1$ when $\tau \rightarrow 0$. (Observe that we already have $t-s \rightarrow 0$ together with $\tau \rightarrow 0$ - however, we do not need it here.) At last, we find for $\tau$ chosen sufficiently small,

$$
\frac{\omega(\tau)}{\tau} \leq \frac{t-s}{\tau} \frac{\tilde{\omega}(t-s)}{t-s} \leq \frac{1}{\cos \omega(\tau)} \sup _{\xi} \frac{\tilde{\omega}(\xi)}{\xi} \leq(1+\varepsilon) \lim _{\xi \rightarrow 0} \frac{\tilde{\omega}(\xi)}{\xi} .
$$

It follows that the leftmost and rightmost limits in (12) exist and are equal to the corresponding limits with respect to arc length. Therefore, it suffices to prove the inequalities involving $\kappa(\mathbf{x})$ for the quantities $\omega$ and $\Omega$ only.

Clearly, $\Omega(\mathbf{n},|\mathbf{x}-\mathbf{y}|) \leq \arccos \langle\mathbf{u}, \mathbf{v}\rangle \leq \omega(\mathbf{n},|\mathbf{x}-\mathbf{y}|)$ for all $\mathbf{u} \in \mathbf{n}(\mathbf{x}), \mathbf{v} \in \mathbf{n}(\mathbf{y})$. Putting $\tau=|\mathbf{y}-\mathbf{x}|$, and recalling that $\mathbf{n}(\mathbf{x})$ is unique by condition of existence of $\kappa(\mathbf{x})$, we obtain

$$
\lim _{\tau \rightarrow 0} \frac{\Omega(\mathbf{n}, \tau)}{\tau} \leq(\kappa(\mathbf{x})=) \lim _{\mathbf{y} \rightarrow \mathbf{x} \in \mathbf{v} \in \mathbf{n}(\mathbf{y})} \frac{\arccos \langle\mathbf{n}(\mathbf{x}), \mathbf{v}\rangle}{|\mathbf{x}-\mathbf{y}|} \leq \lim _{\tau \rightarrow 0} \frac{\omega(\mathbf{n}, \tau)}{\tau} .
$$

In the following proposition arccos will denote the branch with values in $[0, \pi]$. 
Proposition 2. Let $\gamma$ be a closed convex curve, and (7) and (8) be the modulus of continuity and the minimal oscillation of the (in general, multi-valued) unit normal vector function $\mathbf{n}(\mathbf{x})$.

(i) If the curvature exists and is bounded from above by $\kappa_{0}$ all over $\gamma$, then there exists a bound $\tau_{0}>0$ so that for any two points $\mathbf{x}, \mathbf{y} \in \gamma$ with $|\mathbf{x}-\mathbf{y}| \leq \tau \leq \tau_{0}$ we must have $\omega(\mathbf{n}, \tau)<\pi / 2$ and $\arccos \langle\mathbf{n}(\mathbf{x}), \mathbf{n}(\mathbf{y})\rangle \leq \kappa_{0} \tau / \cos (\omega(\mathbf{n}, \tau))$. Thus we also have $\omega(\mathbf{n}, \tau) \leq \kappa_{0} \tau / \cos (\omega(\mathbf{n}, \tau))$ for $\tau \leq \tau_{0}$.

(ii) If the curvature $\kappa(\mathbf{x})$ exists (linearly, that is, according to arc length parametrization) almost everywhere, and is bounded from below by $\kappa_{0}$ (linearly) almost everywhere on $\gamma$, then for any two points $\mathbf{x}, \mathbf{y} \in \gamma$ with $|\mathbf{x}-\mathbf{y}| \geq \tau$ and for all $\mathbf{u} \in \mathbf{n}(\mathbf{x}), \mathbf{v} \in \mathbf{n}(\mathbf{y})$ we have $\arccos \langle\mathbf{u}, \mathbf{v}\rangle \geq \kappa_{0} \tau$ and hence $\Omega(\mathbf{n}, \tau) \geq \kappa_{0} \tau$.

Proof. Consider first $(i)$. In this case $\mathbf{n}$ is a single-valued function. Recall that $\alpha$ stands for the tangent angle function, and so with $\mathbf{x}=\gamma\left(s_{0}\right)$ and $\mathbf{y}=\gamma(t) \arccos \langle\mathbf{n}(\mathbf{x}), \mathbf{n}(\mathbf{y})\rangle=$ $\alpha(t)-\alpha\left(s_{0}\right)$, supposing that on the counterclockwise closed arc xy of $\gamma$ the rotation of the outer unit normal vector is at most $\pi$. (In case of the rotation exceeding $\pi$, the complementary arc must have rotation below $\pi$, and considering the negatively oriented curve, i.e. a reflection of $\gamma$, we can conclude the same way.) Since the curvature is just $\kappa=\alpha^{\prime}$ ( $\alpha$ written in arc length parametrization), by condition $\alpha$ is an everywhere differentiable function (with respect to arc length). Thus we can apply the Lagrange mean value theorem to find some parameter $u \in\left(s_{0}, t\right)$ satisfying

$$
\alpha(t)-\alpha\left(s_{0}\right)=\alpha^{\prime}(u)\left(t-s_{0}\right) .
$$

Now we can apply the condition $\alpha^{\prime}=\kappa \leq \kappa_{0}$ to get

$$
\arccos \langle\mathbf{n}(\mathbf{x}), \mathbf{n}(\mathbf{y})\rangle \leq \kappa_{0}\left(t-s_{0}\right) .
$$

It remains to estimate the arc length $t-s_{0}$ in function of $\tau$.

Let now $\mathbf{x}, \mathbf{y}$ be two arbitrary points of $\gamma$ and consider the counterclockwise arc of $\gamma$ between these points. Let us suppose that this arc has total curvature less than $\pi / 2$. Since $\kappa$ exists and is bounded everywhere by $\kappa_{0}$, a standard compactness argument yields $\omega(\mathbf{n}, \tau)<\pi / 2$ for $\tau:=|\mathbf{y}-\mathbf{x}| \leq \tau_{0}$. As now $\mathbf{n}(\mathbf{x})$ is single-valued, we have $\arg \mathbf{t}(\mathbf{x})=\alpha\left(s_{0}\right)$ with the unique tangent vector at $\mathbf{x}$, and we can write

$$
\begin{aligned}
t-s_{0} & =\int_{s_{0}}^{t} 1 d s \leq \int_{s_{0}}^{t} \frac{\cos \left(\alpha(s)-\alpha\left(s_{0}\right)\right)}{\cos \left(\alpha(t)-\alpha\left(s_{0}\right)\right)} d s=\frac{1}{\cos \left(\alpha(t)-\alpha\left(s_{0}\right)\right)} \int_{s_{0}}^{t}\langle\dot{\gamma}(s) ; \mathbf{t}(\mathbf{x})\rangle d s \\
& \leq \frac{1}{\cos \omega(\mathbf{n},|\mathbf{y}-\mathbf{x}|)}\left\langle\int_{s_{0}}^{t} \dot{\gamma}(s) d s ; \mathbf{t}(\mathbf{x})\right\rangle=\frac{\langle\mathbf{y}-\mathbf{x} ; \mathbf{t}(\mathbf{x})\rangle}{\cos \omega(\mathbf{n}, \tau)} \leq \frac{\tau}{\cos \omega(\mathbf{n}, \tau)}
\end{aligned}
$$

On combining this with (13), the assertion ( $i$ ) follows.

To prove $(i i)$ we still can use that $\alpha$ is a monotonic function, hence is almost everywhere differentiable and, as detailed above, for any $\mathbf{u} \in \mathbf{n}(\mathbf{x}), \mathbf{v} \in \mathbf{n}(\mathbf{y})$ we have

$\arccos \langle\mathbf{u}, \mathbf{v}\rangle=\arg \mathbf{u}-\arg \mathbf{v} \geq \tilde{\mathbf{n}}_{-}(t)-\tilde{\mathbf{n}}_{+}\left(s_{0}\right)=\alpha_{-}(t)-\alpha_{+}\left(s_{0}\right) \geq \int_{s_{0}}^{t} \alpha^{\prime}(s) d s \geq \kappa_{0}\left(t-s_{0}\right)$

by condition of $\kappa=\alpha^{\prime} \geq \kappa_{0}$ (linearly) a.e. on $\gamma$. (As above, we may assume that $\arg \mathbf{u}-\arg \mathbf{v}$ does not exceed $\pi$, as otherwise we may consider the complementary arc, i.e. the reflected curve with respect to the line of $\mathbf{x}$ and $\mathbf{y}$, e.g.) It is obvious that the 
arc length of $\gamma$ between $\mathbf{x}$ and $\mathbf{y}$ is at least the distance of $\mathbf{x}$ and $\mathbf{y}$, hence the assertion follows.

Rotations of $\mathbb{C}=\mathbb{R}^{2}$ about the origin $O$ by the counterclockwise measured (positive) angle $\varphi$ will be denoted by $U_{\varphi}$, that is,

$$
U_{\varphi}=\left(\begin{array}{cc}
\cos \varphi & -\sin \varphi \\
\sin \varphi & \cos \varphi
\end{array}\right)
$$

We denote $T$ the reflection to the $y$-axis, i.e. the linear mapping defined by $\left(\begin{array}{cc}-1 & 0 \\ 0 & 1\end{array}\right)$.

Definition 3 (Mangled $n$-gons). Let $2 \leq k \in \mathbb{N}$ and put $n=4 k-4, \varphi^{*}:=\frac{\pi}{2 k}$. We define the standard mangled $n$-gon as the convex $n$-gon

$$
M_{k}:=\operatorname{con}\left\{A_{1}, \ldots, A_{k-1}, A_{k+1}, \ldots, A_{2 k-1}, A_{2 k+1}, \ldots, A_{3 k-1}, A_{3 k+1}, \ldots, A_{4 k-1}\right\},
$$

of $n=4 k-4$ vertices with

$$
A_{m}:=\left(\sum_{j=1}^{m} \cos \left(j \varphi^{*}\right)-\sum_{\ell=1}^{\lfloor m / k\rfloor} \cos \left(\ell k \varphi^{*}\right), \sum_{j=1}^{m} \sin \left(j \varphi^{*}\right)-\sum_{\ell=1}^{\lfloor m / k\rfloor} \sin \left(\ell k \varphi^{*}\right)\right),
$$

where $m \in\{1, \ldots, 4 k\} \backslash\{k, 2 k, 3 k, 4 k\}$. That is, we consider a regular $4 k$-gon of unit sides, but cut out the middle "cross-shape" (i.e., the union of two rectangles which are the convex hulls of two opposite sides of the regular $4 k$-gon, these pairs of opposite sides being perpendicular to each other) and push together the left over four quadrants (i.e., shift the vertices $A_{\ell k}$ to the position of $A_{\ell k-1}$ consecutively to join the remaining sides of the polygon. Observe that taking $A_{0}:=O$, the same formula (16) is valid also for $A_{0}:=O=A_{4 k}=A_{4 k-1}$ and $A_{\ell k}=A_{\ell k-1}, \ell=1,2,3,4$, showing how the vertices of the regular $4 k$-gon were moved into their new positions.)

Now let $\tau>0, \alpha \in \mathbb{R}, \mathbf{x} \in \mathbb{R}^{2}$ and $\varphi \in(0, \pi / 4]$ be arbitrary. Take $k:=\left\lfloor\frac{\pi}{2 \varphi}\right\rfloor$, so that $\varphi^{*}:=\frac{\pi}{2 k} \geq \varphi$.

Then we write $M(\varphi):=M_{k}$, and, moreover, we also define

$$
M(\mathbf{x}, \alpha, \varphi, \tau):=M\left(\mathbf{x}, \alpha, \varphi^{*}, \tau\right):=U_{\alpha}\left(\tau M_{k}\right)+\mathbf{x},
$$

that is, the copy shifted by $\mathbf{x}$ of the $4 k-4$-gon obtained by dilating $M(\varphi)=M_{k}$ from $O=A_{0}=A_{4 k-1}$ with $\tau$ and rotating it counterclockwise about $O$ by the angle $\alpha$.

E.g. if $\varphi \in(\pi / 6, \pi / 4]$, then $k=2, \varphi^{*}=\pi / 4, n=4$, and $M_{2}$ is just a unit square, its side lines having direction tangents \pm 1 and having its lowest vertex at $O$. It is the left over part, pushed together, of a regular octagon of unit side length, when the middle cross-shape is removed from its middle.

It is easy to see that the inradius $\rho(\varphi)$ and the circumradius $R(\varphi)$ of $M(\varphi)=M\left(\varphi^{*}\right)=$ $M_{k}$ are

$$
\left\{\begin{array}{l}
r(\varphi)=\frac{1}{2}\left\{\cot \frac{\pi}{4 k}-\sqrt{2} \cos \left(\frac{1-(-1)^{k}}{8 k} \pi\right)\right\}, \quad\left(k:=\left\lfloor\frac{\pi}{2 \varphi}\right\rfloor\right), \\
R(\varphi)=\frac{1}{2}\left\{\cot \frac{\pi}{4 k}-1\right\},
\end{array}\right.
$$

respectively.

Similarly to the mangled $n$-gons $M_{k}$, we also define the fattened $n$-gons $F_{k}$.

Definition 4 (Fattened $n$-gons). Let $k \in \mathbb{N}$ and put $n=4 k, \varphi^{*}:=\frac{\pi}{2 k}$. We first define the standard fattened $n$-gon as the convex $n$-gon

$$
F_{k}:=\operatorname{con}\left\{A_{1}, \ldots, A_{k-1}, A_{k}, A_{k+1}, \ldots, A_{4 k-1}, A_{4 k}\right\},
$$


of $n=4 k$ vertices with

$$
A_{m}:=\left(\sum_{j=1}^{m} \cos \left(j \varphi^{*}\right)+\sum_{\ell=0}^{\lfloor m / k\rfloor} \cos \left(\ell k \varphi^{*}\right), \sum_{j=1}^{m} \sin \left(j \varphi^{*}\right)+\sum_{\ell=0}^{\lfloor m / k\rfloor} \sin \left(\ell k \varphi^{*}\right)\right) .
$$

That is, we consider a regular $4 k$-gon, but fatten the middle "cross-shape" to twice as wide, and move the four quadrants to the corners formed by this width-doubled cross (i.e., shift the vertices $A_{\ell k}$ to the position of $A_{\ell k-1}+2\left(A_{\ell k}-A_{\ell k-1}\right)$ consecutively to join the remaining sides of the polygon). Observe that $A_{4 k-1}=(-1,0)$ and $A_{4 k}=(1,0)$.

Let $\tau>0, \alpha \in \mathbb{R}, \mathbf{x} \in \mathbb{R}^{2}$ and $\varphi \in(0, \pi)$ be arbitrary. Now we take $k:=\left\lceil\frac{\pi}{2 \varphi}\right\rceil$, whence $\varphi^{*}:=\frac{\pi}{2 k} \leq \varphi$.

Then we write $F(\varphi):=F_{k}$, and, moreover, we also define

$$
F(\mathbf{x}, \alpha, \varphi, \tau):=F\left(\mathbf{x}, \alpha, \varphi^{*}, \tau\right):=U_{\alpha}\left(\tau F_{k}\right)+\mathbf{x},
$$

that is, the copy shifted by $\mathbf{x}$ of the $4 k$-gon obtained by dilating $F(\varphi)=F_{k}$ from $O$ with $\tau$ and rotating it counterclockwise about $O$ by the angle $\alpha$.

E.g. if $\varphi \geq \pi / 2$, then $k=1, \varphi^{*}=\pi / 2, n=4$, and $F_{4}$ is just the square spanned by the vertices $(1,0),(1,2),(-1,2),(-1,0)$ and having sides of length 2 .

Observe that using the usual Minkowski addition, we can represent the connections of these deformed $n$-gons and the regular $n$-gon easily. Write $Q_{n}$ for the regular $n$-gon placed symmetrically to the $y$-axis but above the $x$-axis with $O \in \partial Q_{n}$ a midpoint (hence not a vertex) of a side of $Q_{n}$. (This position is uniquely determined.) Also, denote the standard square as $S:=Q_{4}:=\operatorname{con}\{(1 / 2,0) ;(1 / 2,1) ;(-1 / 2,1) ;(-1 / 2,0)\}$. Then we have $M_{k}+S=Q_{4 k}$ and $Q_{4 k}+S=F_{k}$.

It is also easy to see that the inradius $\mathfrak{r}(\varphi)$ and the circumradius $\mathfrak{R}(\varphi)$ of $F(\varphi)=F\left(\varphi^{*}\right)$ are

$$
\mathfrak{r}(\varphi)=\frac{1}{2} \cot \frac{\pi}{4 k}+\frac{1}{2} \quad\left(k:=\left\lceil\frac{\pi}{2 \varphi}\right\rceil\right)
$$

and

$$
\mathfrak{R}(\varphi)=\left\{\begin{array}{ll}
\frac{1}{2 \sin \frac{\pi}{4 k}}+\frac{1}{\sqrt{2}} & \text { if } 2 \nmid k \\
\sqrt{\frac{1}{2}+\frac{1}{4 \sin ^{2} \frac{\pi}{4 k}}+\frac{1}{\sqrt{2}} \cot \frac{\pi}{4 k}} & \text { if } 2 \mid k
\end{array} \quad\left(k:=\left\lceil\frac{\pi}{2 \varphi}\right\rceil\right),\right.
$$

respectively.

The actual values of the above in- and circumradii in (18), (22), (23) are not important, but observe that for $\varphi \rightarrow 0$, or, equivalently, for $k \rightarrow \infty$, we have the asymptotic relation $r(\varphi) \sim R(\varphi) \sim \mathfrak{r}(\varphi) \sim \mathfrak{R}(\varphi) \sim \frac{1}{\varphi}$.

\section{The Discrete BlaschKe theorems}

Theorem 3. Let $K \subset \mathbb{C}$ be a convex body and $0<\varphi<\pi / 4$. Denote $\mathbf{n}$ the (multivalued) function of outer unit normal(s) to the closed convex curve $\gamma:=\partial K$ and assume that $\omega(\mathbf{n}, \tau) \leq \varphi<\pi / 4$. Put $k:=\left\lfloor\frac{\pi}{2 \varphi}\right\rfloor$. If $\mathbf{x} \in \partial K=\gamma$, and $\mathbf{n}_{0}=(\sin \alpha,-\cos \alpha) \in \mathbf{n}(\mathbf{x})$ is outer unit normal to $\gamma$ at $\mathbf{x}$, then $M(\mathbf{x}, \alpha, \varphi, \tau) \subset K$.

Proof. Because $\varphi \leq \varphi^{*}:=\pi /(2 k)$ and $M(\mathbf{x}, \alpha, \varphi, \tau)=M\left(\mathbf{x}, \alpha, \varphi^{*}, \tau\right)$, it suffices to present a proof for the case when $\varphi=\varphi^{*}=\frac{\pi}{2 k}$.

Applying simple transformations we may reduce to the case $\mathbf{x}=O$ and $\alpha=0, \tau=1$. With these restrictions we are to prove $M_{k} \subset K$, where $O \in K=\partial \gamma,(0,-1)$ is an outer 
normal to $K$ at $O$, and $\omega(\mathbf{n}, 1) \leq \varphi$. Denote $P=(a, b)$ the first point, along $\gamma$ following $O$ counterclockwise, satisfying that $(1,0)$ is outer normal to $K$ at $P$. Clearly, then $\gamma$ can be parameterized with the $x$-values along the $x$-axis so that $\gamma(x)=(x, g(x))$ for values $x \in[0, a]$, and $g$ is a convex function on $[0, a]$.

Consider $A_{m}=\left(a_{m}, b_{m}\right)$ defined in (16) for $m=0, \ldots, k-1$, putting here $A_{0}:=$ $A_{4 k-1}=O$, and consider the function

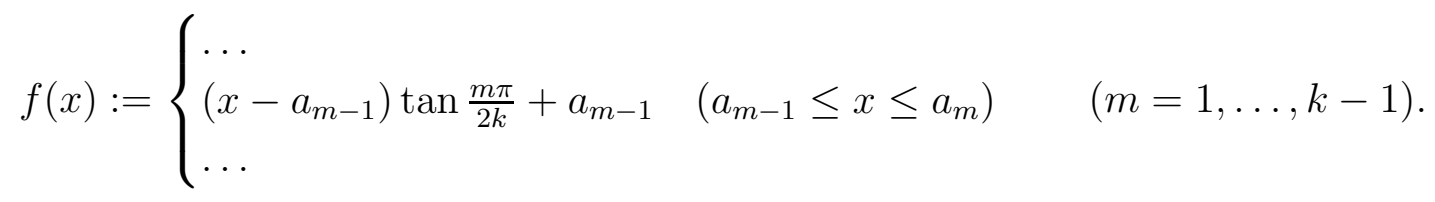

Moreover, denote the broken line joining $O=A_{0}, A_{1}, \ldots, A_{k-1}$ as $L$, that is,

$$
L:=\left\{(x, f(x)): 0 \leq x \leq a_{k-1}\right\} .
$$

Lemma 1. Let $O \in \gamma=\partial K,(0,-1)$ is outer normal to $K$ at $O$, and $\omega(\mathbf{n}, 1) \leq \varphi=\frac{\pi}{2 k}$. With the notations above, we have

(i) $a \geq a_{k-1}=\frac{1}{2}\left(\cot \frac{\pi}{4 k}-1\right)(=R(\varphi))$.

(ii) $0 \leq g(x) \leq f(x)$ for all $x \in\left[0, a_{k-1}\right]$.

(iii) $g_{ \pm}^{\prime}(x) \leq f_{ \pm}^{\prime}(x)$ for all $x \in\left(0, a_{k-1}\right)$ and $g_{+}^{\prime}(0) \leq f_{+}^{\prime}(0), g_{-}^{\prime}\left(a_{k-1}\right) \leq f_{-}^{\prime}\left(a_{k-1}\right)$.

(iv) $b:=g(a) \geq a_{k-1}$.

(v) $L \subset K$.

Proof. Let $a^{*}:=\min \left(a, a_{k-1}\right)$. We argue by induction on $m$, where $m=1, \ldots k-1$, and the inductive assertions will comprise

(i') $a^{*} \geq a_{m}$;

(ii') $0 \leq g(x) \leq f(x)$ for all $x \in\left[a_{m-1}, a_{m}\right]$;

(iii') $g_{ \pm}^{\prime}(x) \leq f_{ \pm}^{\prime}(x)$ for all $x \in\left(a_{m-1}, a_{m}\right)$ and $g_{+}^{\prime}\left(a_{m-1}\right) \leq f_{+}^{\prime}\left(a_{m-1}\right), g_{-}^{\prime}\left(a_{m}\right) \leq f_{-}^{\prime}\left(a_{m}\right)$. Clearly, if we show this for all $m=1, \ldots, k-1$ then (i)-(iii) of the Lemma will be proved.

Let us start with $m=1$. Since $O=(0,0) \in \gamma$, we have $g\left(a_{m-1}\right)=g(0)=0 \leq$ $f\left(a_{m-1}\right)=f(0)=0$. Let $S:=\left\{x \in\left[0, a_{1}\right]:\left.g\right|_{[0, x]} \leq\left. f\right|_{[0, x]}\right\}$. Clearly, $S$ is a (possibly degenerate) closed interval with left end point 0 , say $[0, X]$. Our aim is to prove that $S=\left[0, a_{1}\right]$. Clearly, if $X=a_{1} \in S$, then a relative neighborhood of $X$ belongs to $S$, too. We prove the same thing for any other $X \in S$. Observe that the distance of $O=A_{0}$ and $A_{1}$ is 1 , and all other points of the triangle $\Delta:=\Delta\left(O,\left(a_{1}, 0\right), A_{1}\right)$ are closer than 1 to $O=A_{0}$. In particular, in case $X<a_{1}$, both $\{(x, g(x)): 0 \leq x \leq X\}$ and also a small neighborhood of $(X, g(X)) \in \Delta$ is also closer to $O$ than 1 . It follows that the continuous curve $\gamma$ runs in the 1-neighborhood of $O$ even in an appropriately small neighborhood of $(X, g(X)) \in \gamma$. Therefore, by assumption on the change of the normal to $\gamma$, the vector $(0,1)$ in the counterclockwise taken angular region between the left and right hand side half-tangents (oriented according to the positive orientation of $\gamma$ ) to $\gamma$ at $O$, cannot rotate over $(\cos \varphi, \sin \varphi)$ along $\{(x, g(x)): 0 \leq x \leq X+\eta\}$ for some positive value of $\eta$. That is, $a^{*} \geq X+\eta$ and the representation $\gamma(x)=(x, g(x))$ is valid for $x \in[0, X+\eta]$; moreover, $g_{ \pm}^{\prime}(x) \leq \tan \varphi$ for all $x \in[0, X+\eta]$. In conclusion, $g(x)=\int_{0}^{x} g^{\prime}(\xi) d \xi \leq x \cdot \tan \varphi=f(x)$ for all $x \in[0, X+\eta]$. As a result, we find that $S$ is relatively open. As it is also closed and nonempty, it is the whole interval $\left[0, a_{1}\right]$. This proves (i') and (ii') for $m=1$, and (iii') follows from the fact that $\left\{(x, g(x)): 0 \leq x \leq a_{1}\right\} \subset \Delta$ and thus the distance of any point of $\left\{(x, g(x)): 0 \leq x \leq a_{1}\right\}$ from $O$ is at most 1 .

We proceed by induction. Let $1<m<k$ and assume the assertion for all $m^{\prime}<m$. Then from the inductive hypothesis $a^{*} \geq a_{m-1}, \mu:=\mu_{m-1}:=g_{-}^{\prime}\left(a_{m-1}\right) \leq f_{-}^{\prime}\left(a_{m-1}\right)=$ $\tan ((m-1) \varphi)$ and $g\left(a_{m-1}\right):=y_{m-1} \leq f\left(a_{m-1}\right)=b_{m-1}$. Consider now the function 
$h(x):=y_{m-1}+\left(x-a_{m-1}\right) \tan (m \varphi)$ (defined for $\left.x \in I_{m}:=\left[a_{m-1}, a_{m}\right]\right)$, denote the points $P_{m-1}:=\left(a_{m-1}, y_{m-1}\right)$ and $P_{m}:=\left(a_{m}, h\left(a_{m}\right)\right)$, and define the triangle $\Delta:=\Delta_{m}:=$ $\Delta\left(P_{m-1},\left(a_{m}, y_{m-1}+\mu \cos (m \varphi)\right), P_{m}\right)$. Then $h=\left.f\right|_{I_{m}}-\left(b_{m-1}-y_{m-1}\right) \leq\left. f\right|_{I_{m}}$, and $h_{ \pm}^{\prime}=f_{ \pm}^{\prime}$ on $I_{m}$.

Our aim now is to show that $\gamma$ proceeds inside $\Delta=\Delta_{m}$. Observe that for points $Q$ inside $\Delta$ we have $\left|Q-P_{m-1}\right| \leq 1$, with equality holding only if $Q=P_{m}$. Therefore, for $Q \in \gamma \cap \Delta$ the right half-tangent direction to $\gamma$ cannot exceed $\arctan \mu+\varphi \leq m \varphi$, and, moreover, the same properties hold even for a relative neighborhood of $Q$ on $\gamma$ if $Q \neq P_{m}$.

So we proceed similarly to the case $m=1$. It is obvious that $a^{*}>a_{m-1}$ as $\gamma$ proceeds between slopes $\mu$ and $\tan (m \varphi)$ in the 1-neighborhood of $P_{m-1}$. Take $S:=S_{m}:=\{x \in$ $\left.I_{m}:\left.g\right|_{\left[a_{m-1}, x\right]} \leq\left. h\right|_{\left[a_{m-1}, x\right]}\right\}$. Again, by continuity of $g$ and linearity of $h S$ is a closed interval $\left[a_{m-1}, X\right]$, say. Also, if $X=a_{m}$, then $S=I_{m}$ and so $S$ is relatively open in $I_{m}$, and if $a_{m} \neq X \in S$, then $(X, g(X)) \in \gamma \cap \Delta$ has a small neighborhood where $\gamma$ stays within the 1-neighborhood of $P_{m-1}$, therefore its slope is below $\tan (\arctan \mu+\varphi)$ and $\gamma(x)=(x, g(x))$ extends even until some $X+\eta$; moreover, $a^{*} \geq X+\eta$ and $\mu \leq g_{ \pm}^{\prime} \leq$ $\tan (\arctan \mu+\varphi) \leq \tan (m \varphi)$ holds all over $\left[a_{m-1}, X+\eta\right]$ (where for $a_{m-1}$ and $X+\eta$ we claim only the inequalities for $g_{+}$and $g_{-}$, resp.), proving

$$
\mu\left(x-a_{m-1}\right)+y_{m-1} \leq g(x)=\int_{a_{m-1}}^{x} g^{\prime}(\xi) d \xi+y_{m-1} \leq \tan (m \varphi)\left(x-a_{m-1}\right)+y_{m-1}=h(x)
$$

for all $a_{m-1} \leq x \leq X+\eta$. That is, $\gamma$ stays inside $\Delta$ and $S$ contains a small neighborhood of $X$, too. It follows that $S \neq \emptyset$ is open and closed, while $I_{m}$ is connected, thus $S_{m}=I_{m}$ and (26) holds true even for the whole of $I_{m}$. This proves (i')-(iii'), hence (i)-(iii) of the Lemma.

Applying the above we find $a>a_{k-1}$. However, a simple argument immediately gives also $b>a_{k-1}$, too. Indeed, it suffices to consider the new curve $\widehat{\gamma}:=T\left(U_{-\pi / 2}(\gamma-(a, b))\right.$, obtained from $\gamma$ first shifting it by $-P=-(a, b)$, then rotating it by $-\pi / 2$ about $O$, and finally reflecting it at the $y$-axis. This shows (iv).

Also, applying the Lemma for the reflected curve $\widetilde{\gamma}$ of $\gamma$ with respect to the $y$-axis gives a similar result for the part of $\gamma$ towards the "negative $x$-direction". That is, we find that $\gamma$ joins the points $\widetilde{P}=(\widetilde{a}, \widetilde{b})$ and $P=(a, b)$ with (some of their) outer unit normals $(-1,0)$ and $(1,0)$, respectively, so that the part strictly between these points (and containing $O$ ) does never have horizontal normals, and we have a parametrization $\gamma(x)=(x, g(x))$ for all $\widetilde{a} \leq x \leq a$ with $\widetilde{a} \leq-a_{k-1}, a \geq a_{k-1}$, and $0 \leq g(x) \leq f(|x|),\left|g_{ \pm}^{\prime}(x)\right| \leq f_{ \pm}^{\prime}(|x|)$ for all $x \in\left[-a_{k-1}, a_{k-1}\right]$. Note that we also have $\tilde{b} \geq a_{k-1}$, as above.

Finally let us show $(\mathrm{v})$. Consider any point $(x, f(x))$ of $L$, where $x \in\left[0, a_{k-1}\right]$. There is a vertical line $\ell$ through it that intersects $K$ in a vertical chord $C$ of $K$. The lower endpoint of $C$ is $\left(x, g(x)\right.$. The upper endpoint of $C$ has second coordinate at least $\min \{b, \tilde{b}\} \geq a_{k-1}$. Hence the point $(x, f(x))$ lies on the chord $C$ of $K$, whence in $K$. This proves (v).

Continuation of the proof of Theorem 3. From the above argument - or just reflecting $L$ to the $y$-axis - it is immediate that also the broken line $\widetilde{L}$ joining $A_{3 k+1}, \ldots, A_{4 k-1}=O$ in this order that lies on the boundary of $M_{k}$ belongs to $K$, too. We are left with the upper part joining $A_{k-1}, A_{k+1}, \ldots, A_{2 k-1}, A_{2 k+1}, \ldots, A_{3 k-1}$. Let

$$
L^{+}:=\left[A_{k-1}, A_{k+1}\right] \cup \cdots \cup\left[A_{2 k-2}, A_{2 k-1}\right], \quad L^{-}:=\left[A_{2 k-1}, A_{2 k+1}\right] \cup \cdots \cup\left[A_{3 k-2}, A_{3 k-1}\right] .
$$

Next, let us apply the Lemma to the curve from $P$ onwards in the counterclockwise sense. That is, take $K_{+}:=U_{-\pi / 2}(K-P)$ (with $U_{\alpha}$ as defined in (14)) and $\gamma_{+}:=$ 
$U_{-\pi / 2}(\gamma-P)$ and check that $O \in \gamma_{+}$and also $\gamma_{+}$has an outer normal $(0,-1)$ at $O$; moreover, the same estimate on the modulus of continuity of the normal holds for $\gamma_{+}$. Thus we obtain that $L \subset K_{+}$, that is, $U_{\pi / 2}(L)+P \subset K$. Observe $U_{\pi / 2}(L)=L^{+}-\left(a_{k-1}, a_{k-1}\right)$, which entails $L^{+}+\left(a-a_{k-1}, b-a_{k-1}\right) \subset K$. It suffices to say that $L^{+}+(u, v) \subset K$ with $u, v \geq 0$.

Very similarly (or from this and using reflection) we also obtain $L^{-}+(p, q) \subset K$ with $p \geq 0$ and $q \leq 0$.

We claim that $A_{2 k-1}=\left(0,2 a_{k-1}\right) \in K$. Indeed, $A_{2 k-1}+\left(0,-2 a_{k-1}\right)=O \in K$ and $A_{2 k-1}+(u, v) \in L^{+}+(u, v) \subset K, A_{2 k-1}+(p, q) \in L^{-}+(p, q) \subset K$, and the convex hull of the vectors $\left(0,-2 a_{k-1}\right),(u, v)$ and $(p, q)$ contains $(0,0)$, hence by convexity $A_{2 k-1} \in K$.

Now, for showing $L^{+} \subset K$, recall that $A_{k-1} \in L \subset K, A_{2 k-1} \in K$, and $L^{+}+(u, v) \subset K$. So it remains to see that $L^{+}$is in the convex hull of its two endpoints and the set $L^{+}+(u, v)$ whenever $u, v \geq 0$. Similarly one obtains $L^{-} \subset K$. That concludes the proof.

An even stronger version can be proved considering the modulus of continuity $\tilde{\omega}$ with respect to arc length. We thank this sharpening to Endre Makai, who kindly called our attention to this possibility and suggested the crucial Lemma 2 for the proof.

Theorem 4. Let $K \subset \mathbb{C}$ be a planar convex body and $0<\varphi<\pi / 4$. Denote $\mathbf{n}$ the (multivalued) function of outer unit normal(s) to the closed convex curve $\gamma:=\partial K$ and assume that $\tilde{\omega}(\tau) \leq \varphi<\pi / 4$. Put $k:=\left\lfloor\frac{\pi}{2 \varphi}\right\rfloor$. If $\mathbf{x} \in \partial K=\gamma$, and $\mathbf{n}_{0}=(\sin \alpha,-\cos \alpha) \in$ $\mathbf{n}(\mathbf{x})$ is outer unit normal to $\gamma$ at $\mathbf{x}$, then $M(\mathbf{x}, \alpha, \varphi, \tau) \subset K$.

Proof. We can repeat the argument yielding Theorem 3 with the only change that in the inductive argument for proving Lemma 1, we have to use twice (once for the case $m=1$ to start the inductive argument, and once for general $m$ ) a slightly sharper geometric assertion to ensure that even in this setting the boundary curve $\gamma$ of $K$ will again proceed in the triangles $\Delta:=\Delta\left(O,\left(a_{1}, 0\right), A_{1}\right)$ and $\Delta:=\Delta_{m}:=\Delta\left(P_{m-1},\left(a_{m}, y_{m-1}+\mu \cos (m \varphi)\right), P_{m}\right)$.

The general situation will be covered by the following lemma.

Lemma 2. Let $\Delta=\Delta(P, Q, R)$ be the right- or obtuse triangle spanned by the points $P=(a, p), Q=(b, q)$ with $b>a$ and $q \geq p$, and $R=(b, r)$ with $r>q$. Denote $\rho:=\sqrt{(b-a)^{2}+(r-p)^{2}}$ the length of the longest side of $\Delta$, and let $\mu:=(q-p) /(b-a)$, resp. $\nu:=(r-p) /(b-a)$ be the slopes of sides $P Q$ and $P R$, respectively, with corresponding angles $\psi:=\arctan \mu$ and $\lambda:=\arctan \nu$. Denote $\varphi:=\lambda-\psi$ the angle of $\Delta$ at $P$.

Let $\Gamma$ be a convex curve of arc length $\rho$, connecting the points $P$ and $N=(n, s)$ and having all its tangent vectors at all points of $\Gamma$ (including the right half tangent at $P$ and the left half tangent at $N$ ) with angles between $\psi$ and $\psi+\varphi=\lambda$. Then $n \geq b$, the only possibility for equality is when $N=R$, otherwise $n>b$ and $\Gamma$ intersects the vertical side of $\Delta$ at a mesh point $M=(b, m)$ with $q \leq m<r$. Moreover, $s \in[p+\mu(n-a), r]$.

Proof. By convexity, the non-empty valued, multivalued tangent vector function $\mathbf{t}$ along $\Gamma$ is continuous (in the weak sense) and nondecreasing, and also we have for the multivalued tangent angle function $\widehat{\alpha}(\sigma)=\arg \mathbf{t}(\sigma) \in[\psi, \lambda]$ for all $0 \leq \sigma \leq \rho$, i.e. all along $\Gamma$. Therefore,

$$
(n-a, s-p)=N-P=\int_{0}^{\rho} \mathbf{t}(\sigma) d \sigma=\int_{0}^{\rho}(\cos (\alpha(\sigma)), \sin (\alpha(\sigma)) d \sigma,
$$

now neglecting the linearly 0 -measure set of points where $\mathbf{t}$ or $\widehat{\alpha}$ is indeed multi-valued. By condition we find $n-a \geq \rho \cos \lambda=b-a$ and equality would imply $\cos \alpha(\sigma)=\cos \lambda$ 
a.e., that is $\Gamma=[P, R]$. Otherwise by $|\Gamma|=\rho$ and $n>b$ we surely have $s<r$. Finally, for the directional tangent of the chord $[P, N]$ we see

$$
\frac{s-p}{n-a}=\frac{\int_{0}^{\rho} \sin \alpha(\sigma) d \sigma}{\int_{0}^{\rho} \cos \alpha(\sigma) d \sigma} \geq \frac{\int_{0}^{\rho} \sin \psi d \sigma}{\int_{0}^{\rho} \cos \psi d \sigma}=\tan \psi=\mu .
$$

The Lemma follows.

In applying the above lemma we start with the observation that by condition $\tilde{\omega}(1) \leq \varphi$, the tangent angle of $\gamma$ can increase at most $\varphi$ along the part of $\gamma$ which is closer than 1 to the point $O$ (in case $m=1$ ) or to $P_{m-1}$ (in case of the inductive step with general $m)$. Therefore, proceeding along $\gamma$ with arc length 1 and denoting this arc of $\gamma$ as $\Gamma$, we will have a convex curve, with tangent angles between $\psi$ and $\psi+\varphi$, as in the above lemma. Therefore, Lemma 2 will ensure that the argument goes through for proving the corresponding version of Lemma 1 with $\omega(\mathbf{n}, 1)$ replaced by $\tilde{\omega}(1)$. Otherwise the argument is the same.

Theorem 5. Let $K \subset \mathbb{C}$ be a (planar) convex body and $\tau>0$. Denote $\mathbf{n}$ the (multivalued) function of outer unit normal(s) to the closed convex curve $\gamma:=\partial K$ and assume that $\Omega(\mathbf{n}, \tau) \geq \varphi$. Take $k:=\left\lceil\frac{\pi}{2 \varphi}\right\rceil$. If $\mathbf{x} \in \partial K=\gamma$, and $\mathbf{n}_{0}=(\sin \alpha,-\cos \alpha) \in \mathbf{n}(\mathbf{x})$ is normal to $\gamma$ at $\mathbf{x}$, then $F(\mathbf{x}, \alpha, \varphi, \tau) \supset K$.

Proof. Because $\varphi \geq \varphi^{*}:=\frac{\pi}{2 k}$ and $F(\mathbf{x}, \alpha, \varphi, \tau)=F\left(\mathbf{x}, \alpha, \varphi^{*}, \tau\right)$, it suffices to present a proof for the case when $\varphi=\varphi^{*}=\frac{\pi}{2 k}$.

Applying simple transformations we may reduce to the case $\mathbf{x}=O$ and $\alpha=0, \tau=1$. With these restrictions we are to prove $F_{k} \supset K$, where $O \in K=\partial \gamma,(0,-1)$ is an outer normal to $K$ at $O$, and $\Omega(\mathbf{n}, 1) \geq \varphi$.

Denote $P=(a, b)$ the first point counterclokwise after $O$, along $\gamma$, satisfying that $(1,0)$ is an outer unit normal to $K$ at $P$. Clearly, then $\gamma$ can be parameterized with the $x$-values along the $x$-axis so that $\gamma(x)=(x, g(x))$ for values $x \in[0, a]$, and $g$ is a convex function on $[0, a]$.

Note that in case $a=0$ we necessarily have $K \subset\{(x, y): x \leq 0\}$, and so the degenerate case becomes trivial as regards proving $K \cap\{(x, y): x \geq 0\} \subset F_{k} \cap\{(x, y): x \geq 0\}$. Therefore, we can assume that we have the non-degenerate case.

Similarly to $(20)$, we define $A_{m}=\left(a_{m}, b_{m}\right)$ for $m=0, \ldots, k$ (here $A_{0}:=(0,0)=O$ ), and consider the function

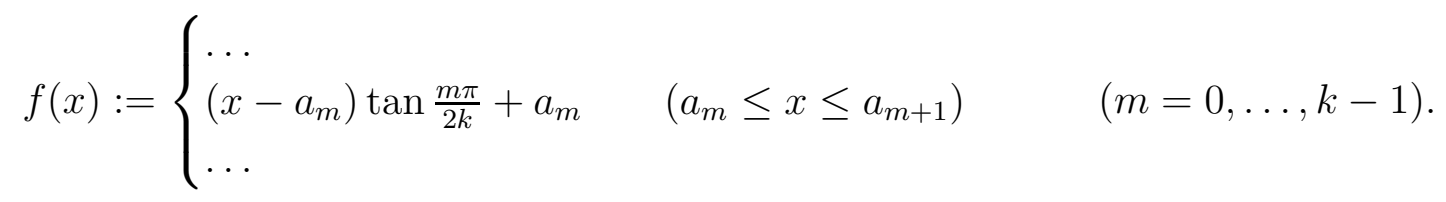

Moreover, now $L$ will denote the broken line joining $O=A_{0}, A_{1}, \ldots, A_{k}, \frac{1}{2}\left(A_{k}+A_{k+1}\right)=$ $\left(a_{k}, a_{k}\right)$ in this order, that is,

$$
L:=\left\{(x, f(x)): 0 \leq x \leq a_{k}\right\} \cup\left[A_{k},\left(a_{k}, a_{k}\right)\right] .
$$

We write

$$
L_{1}:=L \cup\{(x, 0): x \leq 0\} \cup\left\{\left(a_{k}, y\right): y \geq a_{k}\right\} .
$$

Then $\mathbb{R}^{2} \backslash L_{1}$ will have two connected components; the convex one will be denoted by $K_{1}$.

Lemma 3. Let $O \in K=\partial \gamma,(0,-1)$ be an outer normal to $K$ at $O$, and $\Omega(\mathbf{n}, 1) \geq \varphi=\frac{\pi}{2 k}$. With the notations above, we have

(i) $a \leq a_{k}=\frac{1}{2}\left(\cot \frac{\pi}{4 k}+1\right)(=\mathfrak{r}(\varphi))$. 
(ii) $0 \leq f(x) \leq g(x)$ for all for all $x \in[0, a]$.

(iii) $f_{ \pm}^{\prime}(x) \leq g_{ \pm}^{\prime}(x) x \in(0, a)$ and $f_{+}^{\prime}(0) \leq g_{+}^{\prime}(0), f_{-}^{\prime}(a) \leq g_{-}^{\prime}(a)$.

(iv) $b:=g(a) \leq a_{k}$.

(v) $K \subset K_{1}$.

Proof. Since the degenerate case $a=0$ is trivial (observe that (ii) is then undefinied, but cf. the paragraph before (28)), we assume $a>0$.

Let $a^{*}:=\min \left(a, a_{k}\right)$. We argue by induction on $m$, where $m=0, \ldots k-1$, and the inductive assertions will comprise

(i') Either $a \leq a_{m}$ or both(ii') and (iii') hold, where

(ii') $0 \leq f(x) \leq g(x)$ for all $x \in\left[a_{m}, \min \left(a, a_{m+1}\right)\right]$;

(iii') $g_{ \pm}^{\prime}(x) \geq f_{ \pm}^{\prime}(x)$ for all $x \in\left[a_{m}, \min \left(a, a_{m+1}\right)\right]$ (except for $g_{-}^{\prime}(0)$ and also for $g_{+}^{\prime}(a)$ if the second occurs).

Clearly, if we show this for all $m=0, \ldots, k-1$ then (i)-(iii) of the Lemma will be proved.

Let us start with $m=0$. Since $O=(0,0) \in \gamma$, we have $g\left(a_{m}\right)=g(0)=0 \geq f\left(a_{m}\right)=$ $f(0)=0$. Let $S:=\left\{x \in\left[0, a_{1}\right]:\left.g\right|_{[0, x]} \geq\left. f\right|_{[0, x]}\right\}$. Clearly, by continuity of $f$ and $g$, $S$ is a closed interval with left endpoint 0 . Our aim is to prove that $S=[0, \min (a, 1)]$. Indeed, since $\left.f\right|_{[0,1]} \equiv 0$, and as $(0,-1)$ is normal to $K$ at $O$, we must have $g(x) \geq 0$ for all $0 \leq x \leq a$, as stated in (ii'). Moreover, since $g$ is a convex curve, $g_{ \pm}^{\prime}(x) \geq 0=f_{ \pm}^{\prime}(x)$ for all $x \in(0, \min (a, 1))$, and also $g_{+}^{\prime}(0) \geq f_{+}^{\prime}(0)=0$, furthermore, $g_{-}^{\prime}(\min (a, 1)) \geq$ $f_{-}^{\prime}(\min (a, 1))$. It remains to show $g_{+}^{\prime}(1) \geq \tan \varphi=f_{+}^{\prime}(1)$ in case $\min (a, 1)=1$. But in this case either $a=1$, and then $g_{+}^{\prime}(1)$ does not exist (and the case is listed as exceptional in (iii')), or in view of $|O-(1, g(1))| \geq 1$ any point $(x, g(x))$ along $\gamma$ in the counterclockwise sense after $(1, g(1))$ but before $P$ (that is, with $1<x<a$ ) is of distance $>1$ from $O$, hence by condition its any outer normal direction is at least $\varphi$ larger than that of the outer normal $(0,-1)$ of $O$ : it follows that $g_{ \pm}^{\prime}(x) \geq \tan \varphi$ and thus $g_{+}^{\prime}(1) \geq \tan \varphi=f_{+}^{\prime}(1)$.

We proceed by induction. Let $1 \leq m<k$ and assume the assertion for all $0 \leq m^{\prime}<m$. If $\min \left(a, a_{m}\right)=a$, then (i') holds and we have nothing to prove. Let now $a_{m+1}^{*}:=$ $\min \left(a, a_{m+1}\right)$. If $\min \left(a, a_{m}\right)=a_{m}<a$, then by the inductive assumption we must have $g\left(a_{m}\right) \geq f\left(a_{m}\right)$ and $g_{-}^{\prime}\left(a_{m}\right) \geq f_{-}^{\prime}\left(a_{m}\right), g_{+}^{\prime}\left(a_{m}\right) \geq f_{+}^{\prime}\left(a_{m}\right)=\left.\tan (m \varphi) \equiv f_{ \pm}^{\prime}\right|_{\left(a_{m}, a_{m+1}\right)}$. In view of convexity we thus obtain $\left.g_{ \pm}^{\prime}\right|_{\left(a_{m}, a_{m+1}^{*}\right)} \geq g_{+}^{\prime}\left(a_{m}\right) \geq f_{+}^{\prime}\left(a_{m}\right)=\tan (m \varphi) \equiv$ $\left.f_{ \pm}^{\prime}\right|_{\left(a_{m}, a_{m+1}^{*}\right)}$ and by left continuity of the left hand derivative this extends to $g_{-}^{\prime}\left(a_{m+1}^{*}\right) \geq$ $f_{-}^{\prime}\left(a_{m+1}^{*}\right)$, too. Furthermore, if $a_{m+1}^{*}=a_{m+1}$, i.e. $a<a_{m+1}$, then $g_{+}^{\prime}\left(a_{m+1}^{*}\right)=g_{+}(a)$ does not exist (and is listed in (iii') as exceptional). The only case remaining is when $a_{m+1}^{*}=a_{m+1}$, i.e. $a \geq a_{m+1}$. Let first $a=a_{m+1}$. As before, in this case $g_{+}^{\prime}\left(a_{m+1}^{*}\right)=g_{+}^{\prime}(a)$ does not exist and is excepted in (iii'). Let now $a>a_{m+1}$, and consider a small right neighborhood $\left[a_{m+1}, a_{m+1}+\epsilon\right]$ of $a_{m+1}$ which is contained fully in $[0, a)$. Then in this neighborhood the parametrization $\gamma(x)=(x, g(x))$ extends for a small arc of $\gamma$ in the counterclockwise sense from $P_{m+1}:=\left(a_{m+1}, g\left(a_{m+1}\right)\right)$, hence for this arc the condition on $\Omega$ can be applied. (We will use also the notations $P_{0}, P_{1}, \ldots, P_{m}$ defined analogously as $\left.P_{k}:=\left(a_{k}, g\left(a_{k}\right)\right), k=0,1, \ldots, m\right)$.

First we prove that $\left|P_{m}-P_{m+1}\right| \geq 1$, which will also imply $\left|P_{m}-(x, g(x))\right|>1$ for all $x \in\left[a_{m+1}, a_{m+1}+\epsilon\right]$, too. For this purpose consider the line $\ell(x):=P_{m}+(\tan (m \varphi))\left(x-a_{m}\right)$ and let $Q:=Q_{m}:=\left(a_{m+1}, \ell\left(a_{m+1}\right)\right)$. Note that between $a_{m}$ and $a_{m+1}$ the line $\ell$ runs below the curve of $\gamma$, since for any point $x$ between the endpoints $g_{ \pm}^{\prime}(x) \geq f_{ \pm}^{\prime}(x)=$ $\tan (m \varphi)=\ell^{\prime}(x)$, and $g\left(a_{m}\right)=\ell\left(a_{m}\right)$. It follows that $g\left(a_{m+1}\right) \geq \ell\left(a_{m+1}\right)$ and thus $\left|P_{m+1}-P_{m}\right|^{2} \geq\left(a_{m+1}-a_{m}\right)^{2}+\left(\tan (m \varphi) \cdot\left(a_{m+1}-a_{m}\right)\right)^{2}=1$, as stated.

Hence $\left|P_{m}-(x, g(x))\right|>1$ for all $x \in\left[a_{m+1}, a_{m+1}+\epsilon\right]$ holds and the $\Omega$-condition can be applied to get $\arctan g_{ \pm}^{\prime}(x) \geq g_{+}^{\prime}\left(a_{m}\right)+\varphi \geq f_{+}^{\prime}\left(a_{m}\right)+\varphi=(m+1) \varphi=\arctan f_{ \pm}^{\prime}(x)$. 
In view of the right continuity of the right hand derivative, we thus obtain $g_{+}^{\prime}\left(a_{m+1}\right) \geq$ $\tan ((m+1) \varphi)=f_{+}^{\prime}\left(a_{m+1}\right)$, too.

Therefore, in case (i') does not hold, we conclude (iii'). Since in this case we have $f\left(a_{m}\right) \leq g\left(a_{m}\right)$ by the inductive hypothesis, a simple integration using (iii') proves also (ii').

Therefore, the inductive argument for (i')-(iii') concludes and we obtain (i)-(iii) of the Lemma. It remains to show (iv) and (v). To prove (iv), it suffices to consider the curve $\widehat{\gamma}:=T\left(U_{-\pi / 2}(\gamma-(a, b)) \gamma_{1}\right.$ from the proof of Lemma 1 , which will have $\widehat{P}=(b, a)$ while satisfying all our requirements.

Finally, let us prove (v): clearly it suffices to prove int $K \subset K_{1}$. Because at $O K$ has an outer normal $(0,-1)$, we have int $K \subset\{(x, y): y>0\}$. Similarly, as at $P=(a, b) K$ has an outer normal $(1,0)$, in view of Lemma 3 (i) we also have int $K \subset\{(x, y): x<$ $a\} \subset\left\{(x, y): x<a_{k}\right\}$.

In view of int $K \subset\{(x, y): x<a\}$, it remains to show that $(x, y) \in \operatorname{int} K, 0<x<a$ imply $y>f(x)$. However, the part of $\partial K$ above the open segment $(O,(a, 0))$ consists of two open arcs, the lower one being $\{(x, g(x)): 0<x<a\}$. Thus, for $0<x<a$, $(x, y) \in \operatorname{int} K$ we necessarily have $y>g(x) \geq f(x)$, as was to be shown.

Lemma 4. Let $K, L, L_{1}, K_{1}$ as above. Let $L_{1}+(u, v)$ a translate of $L_{1}$ such that int $K \subset$ $K_{1}+(u, v)$. Further, let $u^{\prime} \geq u$ and $v^{\prime} \leq v$. Then also int $K \subset K_{1}+\left(u^{\prime}, v^{\prime}\right)$ holds.

Proof. In fact, we are to prove that $K_{1} \subset K_{1}+(w, z)$, with arbitrary $w \geq 0 \geq z$. (Then this can be applied with $(w, z)=\left(u^{\prime}-u, v^{\prime}-v\right)$ to get $K_{1}+(u, v) \subset\left(K_{1}+(w, z)\right)+(u, v)=$ $K_{1}+\left(u^{\prime}, v^{\prime}\right)$.) Observe that the special cases with one coordinate of the translation vector being zero already suffice, for $K_{1} \subset K_{1}+(w, 0) \subset\left(K_{1}+(0, z)\right)+(0, w)=K_{1}+(w, z)$ gives the general case, too. Also observe that by symmetry of $K_{1}$ to the line $y=-x$, it suffices to prove one such case, e.g. $K_{1} \subset K_{1}+(0, z)$. However, as $K_{1}$ can be defined as the set of points above a function graph, this last inclusion with $z \leq 0$ is evident.

Continuation of the proof of Theorem 5. Recall that $T$ is the reflection on the $y$-axis; let us introduce also $S$ as the reflection on the line $y=a_{k}$.

From the above argument - or just reflecting $L$ to the $y$-axis - it is immediate that we have also $K \subset T K_{1}$.

We are left with the upper part joining $\frac{1}{2}\left(A_{k}+A_{k+1}\right), A_{k+1}, \ldots, A_{3 k}, \frac{1}{2}\left(A_{3 k}+A_{3 k+1}\right)$. Let

$$
\begin{aligned}
& L^{+}:=\left[\frac{A_{k}+A_{k+1}}{2}, A_{k+1}\right] \cup \bigcup_{m=k+1}^{2 k-1}\left[A_{m}, A_{m+1}\right] \cup\left[A_{2 k}, \frac{A_{2 k}+A_{2 k+1}}{2}\right], \\
& L^{-}:=\left[\frac{A_{2 k}+A_{2 k+1}}{2}, A_{2 k+1}\right] \cup \bigcup_{m=2 k+1}^{3 k-1}\left[A_{m}, A_{m+1}\right] \cup\left[A_{3 k}, \frac{A_{3 k}+A_{3 k+1}}{2}\right] .
\end{aligned}
$$

Next, let us apply Lemma 3 to the curve from $P=(a, b)$ onwards to the counterclockwise sense. That is, take $K_{+}:=U_{-\pi / 2}(K-P)$ and $\gamma_{+}:=U_{-\pi / 2}(\gamma-P)$ and check that $O \in \gamma_{+}$and also $\gamma_{+}$has normal $(0,-1)$ at $O$; moreover, the same estimate on the minimal oscillation of the normal holds for $\gamma_{+}$. Thus we obtain that $K \subset S K_{1}+\left(a-a_{k}, b-a_{k}\right) \subset S K_{1}$, where the last inclusion follows from $a, b \leq a_{k}$ and Lemma 4.

Very similarly (or from this and using reflection) we also obtain $K \subset T S K_{1}$. So putting together the four inclusions, we obtain $K \subset K_{1} \cap T K_{1} \cap S K_{1} \cap T S K_{1}=F_{k}$, i.e. $K \subset F_{k}$, and the proof concludes. 


\section{Further CONSEQUENCES}

As the first corollaries, we can immediately deduce the classical Blaschke theorems. We denote by $D(\mathbf{x}, r)$ the closed disc of centre $\mathbf{x}$ and radius $r$.

Proof of Theorem A.. Let $\tau_{0}$ be the bound provided by (i) of Proposition 2. Under the condition, we find (with $\omega(\mathbf{n}, \tau)<\pi / 2$ )

$$
\omega(\mathbf{n}, \tau) \leq \frac{\kappa_{0} \tau}{\cos (\omega(\mathbf{n}, \tau))}=: \varphi(\tau) \quad\left(\tau \leq \tau_{0}\right)
$$

Let us apply Theorem 3 for the boundary point $\mathbf{x} \in \gamma$ with normal vector $\mathbf{n}(\mathbf{x})=$ $(\sin \alpha,-\cos \alpha)$. If necessary, we have to reduce $\tau$ so that the hypothesis $\varphi(\tau) \leq \pi / 4$ should hold. We obtain that the congruent copy $U_{\alpha}\left(\tau M_{k}\right)+\mathbf{x}$ of $\tau M_{k}$ is contained in $K$, where $k=\lfloor\pi / 2 \varphi(\tau)\rfloor$. Note that $U_{\alpha}\left(\tau M_{k}\right)+\mathbf{x} \supset D(\mathbf{z}, \tau r(\varphi(\tau)))$, where $\mathbf{z}=\mathbf{x}-\tau R(\varphi(\tau)) \mathbf{n}(\mathbf{x})$. When $\tau \rightarrow 0$, also $\varphi(\tau) \rightarrow 0$, therefore also $\omega(\mathbf{n}, \tau) \rightarrow 0$ in view of (33), and we see

$$
\lim _{\tau \rightarrow 0}(\tau R(\varphi(\tau)))=\lim _{\tau \rightarrow 0}(\tau r(\varphi(\tau)))=\lim _{\tau \rightarrow 0} \frac{\tau}{\varphi(\tau)}=\lim _{\tau \rightarrow 0} \frac{\cos (\omega(\mathbf{n}, \tau))}{\kappa_{0}}=\frac{1}{\kappa_{0}} .
$$

Note that we have made use of $\omega(\mathbf{n}, \tau) \rightarrow 0$ in the form $\cos (\omega(\mathbf{n}, \tau)) \rightarrow 1$. It follows that $D\left(\mathbf{x}-\frac{1}{\kappa_{0}} \mathbf{n}(\mathbf{x}), \frac{1}{\kappa_{0}}\right) \subset K$, whence the assertion.

Note that in the above proof of Theorem A we did not assume $C^{2}$-boundary, as is usual, but only the existence of curvature and the estimate $\kappa(\mathbf{x}) \leq \kappa_{0}$. So we found the following stronger corollary (still surely well-known).

Corollary 6. Assume that $K \subset \mathbb{R}^{2}$ is a convex domain with boundary curve $\gamma$, that the curvature $\kappa$ exists all over $\gamma$, and that there exists a positive constant $\kappa_{0}>0$ so that $\kappa \leq \kappa_{0}$ everywhere on $\gamma$. Then to all boundary point $\mathbf{x} \in \gamma$ there exists a disk $D_{R}$ of radius $R=1 / \kappa_{0}$, such that $\mathbf{x} \in \partial D_{R}$, and $D_{R} \subset K$.

Similarly, one can deduce also the "dual" Blaschke theorem, i.e. Theorem B, in a similarly strengthened form. In fact, the conditions can be relaxed even further, as was shown by Strantzen, see [3, Lemma 9.11]. Our discrete approach easily implies Strantzen's strengthened version, originally obtained along different lines.

Corollary 7 (Strantzen). Let $K \subset \mathbb{R}^{2}$ be a convex body with boundary curve $\gamma$. Assume that the (linearly) a.e. existing curvature $\kappa$ of $\gamma$ satisfies $\kappa \geq \kappa_{0}$ (linearly) a.e. on $\gamma$. Then to all boundary point $\mathbf{x} \in \gamma$ there exists a disk $D_{R}$ of radius $R=1 / \kappa_{0}$, such that $\mathbf{x} \in \partial D_{R}$, and $K \subset D_{R}$.

Proof. Now we start with (ii) of Proposition 2 to obtain $\Omega(\tau) \geq \kappa_{0} \tau$ for all $\tau$. Put $\varphi:=\varphi(\tau):=\kappa_{0} \tau$. Clearly, when $\tau \rightarrow 0$, then also $\varphi(\tau) \rightarrow 0$ and $k:=\lceil\pi /(2 \varphi(\tau))\rceil \rightarrow \infty$. Take $\mathbf{n}(\mathbf{x})=(\cos \alpha, \sin \alpha)$ and apply Theorem 5 to obtain $U_{\alpha}\left(\tau F_{k}\right)+\mathbf{x} \supset K$ for all $\tau>0$. Observe that $D_{\varphi}:=D((0, \mathfrak{r}(\varphi)), \mathfrak{R}(\varphi)) \supset F_{k}$, hence $U_{\alpha}\left(\tau D_{\varphi}\right)+\mathbf{x} \supset K$. In the limit, since $\mathfrak{r}(\varphi(\tau)) \sim \mathfrak{R}(\varphi(\tau)) \sim 1 /(\varphi(\tau))=1 /\left(\kappa_{0} \tau\right)$, we find $D\left(\mathbf{x}-\left(1 / \kappa_{0}\right) \mathbf{n}, 1 / \kappa_{0}\right) \supset K$, for any $\mathbf{n} \in \mathbf{n}(\mathbf{x})$, that implies the statement.

\section{THE CASE OF HIGHER DIMENSIONAL SPACES}

The Blaschke and Strantzen theorems in $\mathbb{R}^{d}, d \geq 2$ can easily be deduced from the $\mathbb{R}^{2}$ versions. However, it is more difficult to establish $d$-variable analogs of the above discrete Blaschke type theorems. The main reason for this difficulty is that normal vectors need not vary within one plane, when $\mathbf{x}$ varies along a plane curve on $\partial K$. 
In particular, we do not see if it can happen that for some convex body $K \subset \mathbb{R}^{d}$ the minimal oscillation function $\Omega(\mathbf{n}, \tau)$ is relatively large, while on the restriction to some plane $P$ - that is, for $K_{0}:=K \cap P$ - it is almost zero. It can not happen with exactly zero, as then a straight line segment $L$ belongs to $\partial K_{0}$, and the space normals at relative interior points of $L$ define supporting hyperplanes in the space which are valid also for all other relative interior points of $L$, therefore, $\mathbf{n}(\mathbf{x})=\mathbf{n}(\mathbf{y})$ for all points $\mathbf{x}, \mathbf{y} \in \operatorname{relint} L$. But if with a circle arc $C$ of large radius (so of small curvature) we assume $C \subset \partial K_{0}$, and we define a twisting function $\mathbf{n}(\mathbf{x})$ along $C$, then the halfspaces with outer normals $\mathbf{n}(\mathbf{x})$ through $\mathbf{x}$ mesh in a convex set $K$ with nonempty interior: it is then not too difficult to make $\mathrm{K}$ into a convex body with a few other halfspaces. So at least along the arc $C$ the space normals change considerably, while not in $P$. However, it is not clear if a construction can be made with $\Omega(\mathbf{n}, \tau)$ large and $\Omega(\nu, \tau)$ small when considered globally on $K$ and $K_{0}$, resp. (What is missing in the above idea is to guarantee that the space normals do change considerably between any two points of distance $\geq \tau$, and not only between $\tau$-far points of $C$.)

In any case, we present a version, even if somewhat weaker than one would wish, involving the modulus of continuity with respect to chord length. Again, it is unclear if an extension with respect to geodesic distance on $\partial K$, i.e. the natural extension of arc length in $\mathbb{R}^{2}$, can be established. On the other hand, we will be able to formulate our statements in the generality of infinite dimensional spaces. In fact, here it will be more convenient to consider another form of the modulus of continuity, easily defined even in Banach spaces, where distance of the outer unit normals will be measured in the distance of vectors within the dual space (i.e. chord length), and not the geodesic distance (i.e. angle difference) on the surface of the unit ball of the dual space. So we can introduce the next definitions.

Definition 5. Let $\mathbf{v}: H \rightarrow 2^{M} \backslash\{\emptyset\}$, where $H \subset X, M \subset Y$ are sets in the normed vector spaces $X, Y$, respectively. Define

$$
\bar{\omega}(\tau):=\bar{\omega}(\mathbf{v}, \tau):=\sup \left\{\|\mathbf{u}-\mathbf{w}\|_{Y}: \mathbf{x}, \mathbf{y} \in H,\|\mathbf{x}-\mathbf{y}\|_{X} \leq \tau, \mathbf{u} \in \mathbf{v}(\mathbf{x}), \mathbf{w} \in \mathbf{v}(\mathbf{y})\right\}
$$

and

$$
\bar{\Omega}(\tau):=\bar{\Omega}(\mathbf{v}, \tau):=\inf \left\{\|\mathbf{u}-\mathbf{w}\|_{Y}: \mathbf{x}, \mathbf{y} \in H,\|\mathbf{x}-\mathbf{y}\|_{X} \geq \tau, \mathbf{u} \in \mathbf{v}(\mathbf{x}), \mathbf{w} \in \mathbf{v}(\mathbf{y})\right\} .
$$

In our use of the notion, we will take for $H$ either $\partial K$ or $\partial K \cap P$, equipped with the norm distance from $X$, and $M$ will be the unit ball in the dual space $X^{*}$. In fact, due to the geometrical nature of our subject, we will also need orthogonality, that is, Hilbert space structure, in the estimation of Proposition 8 below.

The main reason to use this type of distance in measuring the change of the outer unit normal vectors is that angles can not be handled elegantly when an angle is multiplied by some scalar (like $2 R / r$ below), for the arising arcsin etc. functions have restricted domain. On the other hand, increase of distance estimates are naturally and easily formulated.

Recall that any infinite dimensional real Hausdorff topological linear space we mean by a convex body a bounded closed convex set with non-empty interior. Let $K$ be a convex body in an infinite dimensional real Hilbert space $X$. We have $K=\overline{\operatorname{int} K}$ (this holding in any Hausdorff topological vector space, [4, I, p. 413, Theorem 1, (c)]. We say that $K$ is a smooth convex body if for any $\mathbf{x} \in \partial K$ there exists exactly one unit vector $\mathbf{n}(\mathbf{x})$, called outer unit normal of $K$ at $\mathbf{x}$, such that $K \subset\{\mathbf{y} \in X \mid\langle\mathbf{y}, \mathbf{n}(\mathbf{x})\rangle \leq\langle\mathbf{x}, \mathbf{n}(\mathbf{x})\rangle\}$. Observe that at least one such vector exists, since $\mathbf{x} \notin \operatorname{int} K \neq \emptyset$ (and in a Hausdorff topological vector space a non-empty open convex set and a convex set disjoint to it can be separated by a non-zero continuous linear functional, cf. [4, I, p. 417, Theorem 8].) 
For $\mathbf{c} \in \mathbb{R}^{d}$ and $r>0$ we let $B(\mathbf{c}, r)$ the closed ball of centre $\mathbf{c}$ and radius $r$.

Proposition 8. Let $K \subset H$ be a convex body in the Hilbert space $H$. Assume that $\mathbf{c} \in K$ with $B(\mathbf{c}, r) \subset K \subset B(\mathbf{c}, R)$, where $0<r<R$. Take any two-dimensional plane $P$ through $\mathbf{c}$, denote $K_{0}:=K \cap P \subset P$, and denote $\nu(\mathbf{x})$ the (in general multivalued) outer unit normal vector function of $K_{0}$ at $\mathbf{x} \in \partial K_{0}$ within $P$.

Let $\bar{\omega}(\mathbf{n}, \tau)$ and $\bar{\omega}(\nu, \tau)$ stand for the modulus of continuity (34) of the surface normal vectors $\mathbf{n}$ of $K$ along $\partial K$ in $H$ and $\nu$ of $K_{0}$ along $\partial K_{0}$ in $P \subset H$, respectively. We then have

$$
\bar{\omega}(\nu, \tau) \leq \frac{2 R}{r} \bar{\omega}(\mathbf{n}, \tau)
$$

The following lemmas are well-known elementary facts of space geometry.

Lemma 5. Let $K \subset H$ be a convex body in the Hilbert space $H$, let $\mathbf{x} \in \partial K$ and $P$ be any affine subspace through the point $\mathbf{x}$ and containing some interior point of $K$, too (so that, in particular, $P$ has dimension at least 1). Assume that $P$ is closed (which is satisfied in any case if $\operatorname{dim} P<\infty)$ and denote $\Pi:=\Pi_{P}$ the orthogonal projection of vectors to the affine subspace $P$. Then for any $\mathbf{u} \in \mathbf{n}(\mathbf{x})$ we have $\Pi \mathbf{u} \neq \mathbf{0}$.

Proof. Let $\mathbf{u} \in \mathbf{n}(\mathbf{x})$ be an outer unit normal vector to $K$ at $\mathbf{x}$. In order to prove $\Pi \mathbf{u} \neq \mathbf{0}$, it suffices to take any $\mathbf{y} \in \operatorname{int} K \cap P$ (which exists by assumption), and show that the vector $\mathbf{y}-\mathbf{x}$ is not orthogonal to $\mathbf{u}$. Indeed, for a small ball $B=B(\mathbf{0}, \delta)$ such that $\mathbf{y}+B \subset \operatorname{int} K,\langle\mathbf{u}, \mathbf{y}+\mathbf{b}-\mathbf{x}\rangle \leq 0$ for all $\mathbf{b} \in B$ (because $\mathbf{u} \in \mathbf{n}(\mathbf{x}))$ and $\langle\mathbf{u}, \mathbf{y}-\mathbf{x}\rangle=0$ would imply $\langle\mathbf{u}, \mathbf{b}\rangle \leq 0$ for the whole ball $B$, hence $\langle\mathbf{u}, \mathbf{b}\rangle=0(\forall \mathbf{b} \in B)$ and $\mathbf{u}=\mathbf{0}$, a contradiction.

Lemma 6. Let $K \subset H$ be a convex body in a Hilbert space $H, \mathbf{x} \in \partial K$ and $P$ be any closed affine subspace through the point $\mathbf{x}$ and containing some interior point of $K$, too. Denote $\Pi:=\Pi_{P}$ the orthogonal projection of vectors to $P$ and write $\nu(\mathbf{x})$ for the nonempty set of all outer unit normals to $K_{0}$ in $P$ at $\mathbf{x}$.

Then for arbitrary $\mathbf{w} \in \nu(\mathbf{x})$ there exists some $\mathbf{u} \in \mathbf{n}(\mathbf{x})$ such that $\mathbf{0} \neq \Pi \mathbf{u} \| \mathbf{w}$. Conversely, if $\mathbf{u} \in \mathbf{n}(\mathbf{x})$ then $\Pi \mathbf{u} \neq \mathbf{0}$ and the unit vector $\mathbf{w}:=\Pi \mathbf{u} /|\Pi \mathbf{u}|$ belongs to $\nu(\mathbf{x})$.

Proof. Let now $\mathbf{w} \in \nu(\mathbf{x})$. Consider the orthogonal complement $V$ of $\mathbf{w}$ in $P-\mathbf{x}$, i.e. $V:=\{\mathbf{v} \in P-\mathbf{x}:\langle\mathbf{w}, \mathbf{v}\rangle=0\}$, take $V_{0}:=V \cap B(\mathbf{0}, 1)$, and consider the new convex set $M$ generated as the convex combination of $V_{0}+\mathbf{x}(\subset P)$ and $K$, i.e. $M:=$ $\left\{t \mathbf{z}+(1-t) \mathbf{y}: \mathbf{z} \in K, \mathbf{y} \in V_{0}+\mathbf{x}, 0 \leq t \leq 1\right\}$. Note that clM is a convex body. Clearly $M_{0}:=M \cap P=\left\{t \mathbf{z}+(1-t) \mathbf{y}: \mathbf{z} \in K_{0}, \mathbf{y} \in V_{0}+\mathbf{x}, 0 \leq t \leq 1\right\}$. Observe that $\mathbf{w}$ is still an outer unit normal vector in $P$ at $\mathbf{x}$ even to $M_{0}$.

Consider the disjoint nonempty convex sets int $M-\mathbf{x}$ and $[\mathbf{0}, \mathbf{w}]$. Note that int $M \supset$ $\operatorname{int} K \neq \emptyset$ is open. It follows that there exists a normalized linear functional, whence a unit vector $\mathbf{u}$, such that $\langle\mathbf{u}, \mathbf{y}-\mathbf{x}\rangle<0 \leq\langle\mathbf{u}, \mathbf{w}\rangle$ for all $\mathbf{y} \in \operatorname{int} M$. Obviously by convexity of $M$ and in view of int $M \neq \emptyset$, we have $M \subset \operatorname{cl}$ int $M$. Therefore we find $\langle\mathbf{u}, \mathbf{y}-\mathbf{x}\rangle \leq 0 \leq\langle\mathbf{u}, \mathbf{w}\rangle$ for all $\mathbf{y} \in M$, hence also for vectors in $K$. So clearly any such $\mathbf{u}$ belongs to $\mathbf{n}(\mathbf{x})$. Note that by Lemma $5 \Pi \mathbf{u} \neq \mathbf{0}$.

Moreover, $\langle\mathbf{u},(\mathbf{x}+\mathbf{v})-\mathbf{x}\rangle \leq 0$ for all $\mathbf{v} \in V_{0}$, so that $\mathbf{u} \perp V$. Therefore, $\Pi \mathbf{u} \perp V$ and thus $\Pi \mathbf{u} \| \mathbf{w}$, as needed.

Finally, consider the converse: let $\mathbf{u} \in \mathbf{n}(\mathbf{x})$ ( so that $\Pi \mathbf{u} \neq \mathbf{0}$ ) and write $\mathbf{u}=\Pi \mathbf{u}+\mathbf{u}^{\prime}$, where $\mathbf{u}^{\prime} \perp P$ (i.e., $\left.\mathbf{u} \perp(P-\mathbf{x})\right)$. Clearly, $\left\langle\mathbf{u}^{\prime}, \mathbf{y}-\mathbf{x}\right\rangle=0$ for all $\mathbf{y} \in K_{0} \subset P$. On the other hand $\langle\mathbf{u}, \mathbf{y}-\mathbf{x}\rangle \leq 0$ for all $\mathbf{y} \in K$ (because $\mathbf{u} \in \mathbf{n}(\mathbf{x})$ ), hence for all $\mathbf{y} \in K_{0}$, so combining these two we get $\langle\Pi \mathbf{u}, \mathbf{y}-\mathbf{x}\rangle \leq 0$ for all $\mathbf{y} \in K_{0}$. So we can take $\mathbf{w}:=\Pi \mathbf{u} /|\Pi \mathbf{u}|$, which satisfies $\langle\mathbf{w}, \mathbf{y}-\mathbf{x}\rangle \leq 0$ for all $\mathbf{y} \in K_{0}$, whence $\mathbf{w} \in \nu(\mathbf{x})$ and the assertion follows. 
Lemma 7. Let $K \subset H$ be a convex body in the Hilbert space $H$, and $\mathbf{c} \in \operatorname{int} K$ satisfying $B(\mathbf{c}, r) \subset K \subset B(\mathbf{c}, R)$ with some $0<r<R<\infty$. Let $\mathbf{x}, \mathbf{y} \in \partial K$. Denote $K_{0}:=K \cap P$, where $P$ is a closed affine subspace containing the points $\mathbf{x}, \mathbf{y}$, and $\mathbf{c}$. (Note that $P$ is at least 1 dimensional.) Let us write $\Pi_{P}:=\Pi$ for the orthogonal projection of vectors to $P$.

Denote the nonempty set of all outer unit normals to $K_{0}$ in $P$ at $\mathbf{x}$ and $\mathbf{y}$ as $\nu(\mathbf{x})$ and $\nu(\mathbf{y})$, respectively, and let $\mathbf{w} \in \nu(\mathbf{x}), \mathbf{z} \in \nu(\mathbf{y})$ be arbitrary. Let $\mathbf{u}, \mathbf{v}$ be outer unit normal vectors from $\mathbf{n}(\mathbf{x}), \mathbf{n}(\mathbf{y}) \subset S_{H}$, respectively, such that $\mathbf{0} \neq \Pi \mathbf{u}\|\mathbf{w}, \mathbf{0} \neq \Pi \mathbf{v}\| \mathbf{z}$ (which exist according to Lemma 6). Then we have

$$
|\mathbf{w}-\mathbf{z}| \leq \frac{2 R}{r}|\mathbf{v}-\mathbf{u}|
$$

Consider now the angles $\varphi(\mathbf{u}, \mathbf{v}):=\arccos \langle\mathbf{u}, \mathbf{v}\rangle \in[0, \pi]$ and $\psi(\mathbf{w}, \mathbf{z}):=\arccos \langle\mathbf{w}, \mathbf{z}\rangle \in$ $[0, \pi]$ between these normals. Moreover, assume $\varphi(\mathbf{u}, \mathbf{v})<2 \arcsin \frac{r}{2 R}$. We then have

$$
\psi(\mathbf{w}, \mathbf{z}) \leq 2 \arcsin \left(\frac{2 R}{r} \sin \frac{\varphi(\mathbf{u}, \mathbf{v})}{2}\right) .
$$

Proof. Denote $Z:=Z(\mathbf{x}):=\mathbf{u}^{\perp}+\mathbf{x} . Z$ being a supporting affine hyperplane, int $K \cap Z=$ $\emptyset$, thus the projection $\mathbf{c}^{\prime}$ of $\mathbf{c}$ to $Z$ does not belong to int $K$. That is, $\left|\mathbf{c}^{\prime}-\mathbf{c}\right| \geq r$ in view of $B(\mathbf{c}, r) \subset K$. On the other hand, $\mathbf{c}, \mathbf{x} \in K$ and $|\mathbf{x}-\mathbf{c}| \leq R$, hence reading from the right angle triangle of $\mathbf{c}^{\prime} \mathbf{c x}$, the angle $\Phi$ between the vectors $\mathbf{x}-\mathbf{c}$ and $\mathbf{c}^{\prime}-\mathbf{c} \| \mathbf{u}$ is $\Phi=$ $\arccos (\cos \Phi) \leq \arccos (r / R)$. (In case $\mathbf{c}^{\prime}=\mathbf{x}$, we also have $\Phi=0$.) From this we estimate $|\Pi \mathbf{u}|$. As $\mathbf{x}, \mathbf{c} \in P$, we clearly have $|\Pi \mathbf{u}|=\cos \angle(P, \mathbf{u}) \geq\left\langle\mathbf{u}, \frac{\mathbf{x}-\mathbf{c}}{|\mathbf{x}-\mathbf{c}|}\right\rangle=\cos \Phi \geq r / R$.

Observe that by definition $\mathbf{w}=\Pi \mathbf{u} /|\Pi \mathbf{u}|$ and $\mathbf{z}=\Pi \mathbf{v} /|\Pi \mathbf{v}|$. Therefore, we obtain

$$
\begin{aligned}
|\mathbf{w}-\mathbf{z}| & =\left|\frac{\Pi \mathbf{u}}{|\Pi \mathbf{u}|}-\frac{\Pi \mathbf{v}}{|\Pi \mathbf{v}|}\right|=\left|\frac{\Pi \mathbf{u}-\Pi \mathbf{v}}{|\Pi \mathbf{u}|}+\frac{|\Pi \mathbf{v}|-|\Pi \mathbf{u}|}{|\Pi \mathbf{u}|} \frac{\Pi \mathbf{v}}{|\Pi \mathbf{v}|}\right| \\
& \leq \frac{2|\Pi(\mathbf{v}-\mathbf{u})|}{|\Pi \mathbf{u}|} \leq \frac{2|\mathbf{v}-\mathbf{u}|}{|\Pi \mathbf{u}|} \leq \frac{2 R}{r}|\mathbf{v}-\mathbf{u}|
\end{aligned}
$$

proving (37). Clearly $|\mathbf{u}-\mathbf{v}|=2 \sin (\varphi(\mathbf{u}, \mathbf{v}) / 2)$, so in case $\varphi(\mathbf{u}, \mathbf{v})<2 \arcsin \frac{r}{2 R}$ the right hand side does not exceed 1 and this yields

$$
\psi(\mathbf{w}, \mathbf{z})=2 \arcsin \left(\frac{|\mathbf{w}-\mathbf{z}|}{2}\right) \leq 2 \arcsin \left(\frac{2 R \sin (\varphi(\mathbf{u}, \mathbf{v}) / 2)}{r}\right),
$$

and (38) is proved, too.

As said above, under some restrictions we can derive certain estimates between the modulus of continuity (7) (with respect to chord length) of outer unit normal vectors in the whole space and in a plane section.

Proposition 9. Let $K \subset H$ be a convex body, where $H$ is a Hilbert space. Assume that $\mathbf{c} \in K$ with $B(\mathbf{c}, r) \subset K \subset B(\mathbf{c}, R)$ with $0<r<R \leq \infty$. Take any two-dimensional plane $P$ through $\mathbf{c}$, denote $K_{0}:=K \cap P \subset P$, and denote $\nu(\mathbf{x})$ the (in general multivalued) outer unit normal vector function of $K_{0}$ at $\mathbf{x} \in \partial K_{0}$ within $P$. Let $\omega(\mathbf{n}, \tau)$ and $\omega(\nu, \tau)$ stand for the modulus of continuity (7) (with respect to chord length) of the surface normal vectors $\mathbf{n}$ of $K$ along $\partial K \subset H$ and $\nu$ of $K_{0}$ along $\partial K_{0}$ in $P$, respectively. We then have

$$
\omega(\nu, \tau) \leq 2 \arcsin \left(\frac{2 R}{r} \sin \frac{\omega(\mathbf{n}, \tau)}{2}\right) \quad \text { whenever } \tau<\tau_{0},
$$

where $\tau_{0}$ is chosen to satisfy $0<\tau_{0} \leq 2 r$ and $\omega\left(\mathbf{n}, \tau_{0}\right) \leq 2 \arcsin \frac{r}{2 R}$. 
Remark 1. Note that $K \subset B(\mathbf{c}, R)$ holds with $R:=\operatorname{diam} K-r<\operatorname{diam} K$, always. On the other hand for a symmetric convex body $K \subset H \quad r=w(K) / 2$, where $w(K)$ is the minimal width of $K$, and in general by Steinhagen's Inequality, $\frac{1}{2 \sqrt{d}} w(K) \leq r \leq w(K) / 2$ (if $d$ is odd) and $\frac{\sqrt{d+2}}{2 d+2} w(K) \leq r \leq w(K) / 2$ (if $d$ is even) for $K \subset \mathbb{R}^{d}$ any convex body, see $[2]$.

Proof. Let $P$ be any such plane, and $\mathbf{x}, \mathbf{y} \in \partial K_{0}$ satisfying $|\mathbf{y}-\mathbf{x}| \leq \tau<\tau_{0}$. By condition, for any $\mathbf{u} \in \mathbf{n}(\mathbf{x}), \mathbf{v} \in \mathbf{n}(\mathbf{y})$ we have $\arccos \langle\mathbf{u}, \mathbf{v}\rangle \leq \omega(\mathbf{n}, \tau) \leq \omega\left(\mathbf{n}, \tau_{0}\right) \leq 2 \arcsin \frac{r}{2 R}$ and Lemma 7 applies. Using the vectors $\mathbf{u}, \mathbf{v}$, provided by Lemma 6 to $\mathbf{w}, \mathbf{z}$, and applying also $\arccos \langle\mathbf{u}, \mathbf{v}\rangle \leq \omega(\mathbf{n}, \tau)$ and Lemma 7 , we infer for arbitrary $\mathbf{w} \in \nu(\mathbf{x}), \mathbf{z} \in \nu(\mathbf{y})$ the estimate

$$
\arccos \langle\mathbf{w}, \mathbf{z}\rangle \leq 2 \arcsin \left(\frac{2 R \sin \frac{\arccos \langle\mathbf{u}, \mathbf{v}\rangle}{2}}{r}\right) \leq 2 \arcsin \left(\frac{2 R}{2} \sin \frac{\omega(\mathbf{n}, \tau)}{r}\right) .
$$

Taking supremum over $\mathbf{w} \in \nu(\mathbf{x})$ and $\mathbf{z} \in \nu(\mathbf{y})$ we arrive at (41), whence the assertion.

Proof of Proposition 8. Quite the same way as above, a direct application of Lemma 7, (37) yields the less restricted variant formulated above as Proposition 8.

Even if not sharp in the sense that it does not imply the well-known Blaschke theorem in $\mathbb{R}^{d}$ (but only with the loss of a constant factor, we can still formulate a space version of the discrete Blaschke type theorem above. Namely, we obtain by an application of Proposition 9 and Theorem 3 the following.

Corollary 10. Let $K \subset H$ be a convex body, where $H$ is a Hilbert space. Assume that $\mathbf{c} \in K$ with $B(\mathbf{c}, r) \subset K \subset B(\mathbf{c}, R)$ with $0<r<R \leq \infty$. Let us also assume that with certain parameters $0<\tau<2 r$ and $0<\varphi<2 \arcsin (r /(4 \sqrt{2} R))$, the modulus of continuity (7) of the non-empty valued, multivalued outer unit normal vector function $\mathbf{n}(\mathbf{x})$ satisfies $\omega(\mathbf{n}, \tau) \leq \varphi$.

Then for any boundary point $\mathbf{x} \in \partial K$, any outer unit normal vector $\mathbf{u} \in \mathbf{n}(\mathbf{x})$ and any two-dimensional affine plane $P$ passing through $\mathbf{x}$ and $\mathbf{c}$ the orthogonal projection $\Pi_{P} \mathbf{u}$ of $\mathbf{u}$ to $P$ does not vanish, and $\mathbf{w}:=\Pi_{P} \mathbf{u} /\left|\Pi_{P} \mathbf{u}\right|$ is well-defined. Let us take an arbitrary coordinate system in $P$ and let us put $\alpha:=\arg (\mathbf{w})+\pi / 2 \bmod 2 \pi$ with respect to this coordinate system. Then with the mangled $n-$ gon $M(\mathbf{x}, \alpha, \Phi, \tau)$ defined in Definition 3, (17), we have $M(\mathbf{x}, \alpha, \Phi, \tau) \subset K$, where $\Phi=2 \arcsin \left(\frac{2 R}{r} \sin \frac{\varphi}{2}\right)$.

Proof. Let $\mathbf{x}, \mathbf{c}, P, \mathbf{u}, \mathbf{w}, \tau, \varphi, r, R$ as above. Also let $K_{0}:=K \cap P$ and $\nu(\mathbf{x})$ be the planar outer unit normal vector(s) function of $K_{0}$ in $P$. By the final assertion of Lemma 6 we indeed have $\Pi_{P} \mathbf{u} \neq \mathbf{0}$, moreover, $\mathbf{w} \in \nu(\mathbf{x})$.

Let us estimate the modulus of continuity $\omega(\nu, \cdot)$ of $\nu(\mathbf{x})$. By Proposition 9 we have $\omega(\nu, t) \leq 2 \arcsin \left(\frac{2 R}{r} \sin \frac{\omega(\mathbf{n}, t)}{2}\right)$, whenever $t \leq t_{0}$ where $0<t_{0}<2 r$ is such that $\omega\left(\mathbf{n}, t_{0}\right) \leq$ $2 \arcsin \frac{r}{2 R}$. Let us choose here $t_{0}:=\tau$ : certainly $\tau<2 r$, and by condition $\omega(\mathbf{n}, \tau)=$ $\varphi<2 \arcsin (r /(4 \sqrt{2} R))<2 \arcsin (r /(2 R))$, so the above inequality from Proposition 9 applies even to $\tau$ in place of $t$. We thus find $\omega(\nu, \tau) \leq 2 \arcsin \left(\frac{2 R}{r} \sin \frac{\omega(\mathbf{n}, \tau)}{2}\right) \leq$ $2 \arcsin \left(\frac{2 R}{r} \sin \frac{\varphi}{2}\right)<2 \arcsin \left(\frac{1}{2 \sqrt{2}}\right)<\pi / 4$ as $2 \sigma \leq \arcsin (2 \sin \sigma)$ for $0 \leq \sigma \leq \pi / 4$.

So now we know that the modulus of continuity of the outer unit normal vector(s) function $\nu(\mathbf{x})$ of $K_{0}$ in $P$ satisfies $\omega(\mathbf{n}, \tau) \leq \Phi$ with $\Phi:=2 \arcsin \left(\frac{2 R}{r} \sin \frac{\varphi}{2}\right)<\pi / 4$. 
That is, Theorem 3 applies with $\tau$ and $\Phi$ and the outer unit normal $\mathbf{w} \in \nu(\mathbf{x})$. Thus we are led to $M(\mathbf{x}, \alpha, \Phi, \tau) \subset K$, and the assertion follows.

Remark 2. Corollary 10 provides an inscribed body through description of its plane sections. However, note that in general the inscribed body is not a rotationally symmetric body, the possible axes of symmetry, $\mathbf{w} \in \nu(\mathbf{x})$, varying from plane section to plane section. Nevertheless, in the limit, when curvature exists and $\tau$, whence $\varphi$ tends to 0 , one can deduce a Blaschke type theorem with some ball of radius $\rho=r /\left(2 R \kappa_{0}\right)$ inscribed in $K$ and containing $\mathbf{x} \in \partial K$, as above in Corollary 6.

\section{ACKNOWLEDBEMEnT}

This paper was thoroughly checked by Endre Makai, who gave numerous suggestions for improving the presentation. We thank to him also the sharpening of the inscribed mangled $n$-gon result, by means of the modulus of continuity with respect to arc length, as given above in Theorem 4 .

\section{REFERENCES}

[1] W. Blaschke, Kreis und Kugel, Zweite Auflage, Walter de Gruyter AG, Berlin, 1956.

[2] T. Bonnesen, W. Fenchel, Theory of convex bodies, (Translated from the German and edited by L. Boron, C. Christenson and B. Smith), BCS Associates, Moscow, ID, 1987. x+172 pp

[3] J. N. Brooks, J. B. Strantzen, Blaschke's rolling ball theorem in $\mathbb{R}^{n}$, Mem. Amer. Math. Soc. 80, \# 405, American Mathematical Society, 1989.

[4] N. Dunford, J. T. Schwartz, Linear Operators I, II, III, Interscience - John Wiley \& Sons, New York, London, Second Printing, 1964.

[5] Sz. Gy. RÉvÉsz, On a paper of Erőd and Turán-Markov inequalities for non-flat convex domains, East J. Approx. 12 No. 4 (2006), 451-467

[6] R. Webster, Convexity, Oxford University Press, Oxford, 1994.

A. RÉnyi Institute of Mathematics

Hungarian ACADEmy of ScIEnCES,

Budapest, P.O.B. 127, 1364

HUNGARY

E-mail address: revesz@renyi.hu 